\title{
Beitrag zur Kenntnis der Sumatranischen Dipteren
}

\author{
VON
}

\author{
J. G. H. DE MEIJERE (Amsterdam).
}

Mit Tafel II.

\begin{abstract}
Obgleich Herr Edw. Jacobson auch in den letzten Jahren ausgedehntes Material in unserem ostindischen Archipel zusammengebracht hat, wurde doch nur weniges den heutigen Gefahren der Übersendung ausgestellt. In der vor einiger Zeit eingetroffenen Sendung befand sich auch eine grössere Anzahl Dipteren, jetzt nicht von Java, dem früheren Hauptsammelgebiet dieses nun wohl in der Entomologenwelt sehr bekannten Herren, sondern von Sumatra. Von dieser Insel sind unsere Kenntnisse, was die Dipteren anlangt, noch sehr lückenhaft. Über eine frühere Sendung aus Sumatra habe ich schon in meinen Studien über südostasiatische Dipteren. X, Tijdschr. v. Entomol. LVIII, 1915, p. 64-9\%, berichtet. Das jetzt vorliegende Material stammt aus verschiedenen Gegenden Sumatra's. Es enthält eine bedeutende Anzahl mir unbekannter Stücke, leider vieles in einzelnen Exemplaren. Für eine vollständige Bearbeitung ist es deswegen nicht sehr geeignet; ich berichte hier nư über einen Teil; das übrige bleibe vorläufig aufbewahrt, zumal doch aus diesen Gegenden noch weiteres zu erwarten ist.

Was die öfters genannten Fundorte anlangt, so liegt Air Njuruk, Dempu in Palembang, in 1400 M. Höhe; Suban Ajam und Rimbo Pengadang in Bengkulen; Muara Sako, Sungai Kumbang in Korintji; der „Piek”. von Korintji ebendort; an letzterem wurde in ca. 1700 M. gesammelt. Das manches aus dem Gebirge stammt, erhöht den Wert des Materials bedeutend.

Für die Literatur sei auf mein allgemeines Verzeichnis über meine Studien (Studien XIV, Tijdschr. v. Entom. LX, 1918, p. 275-369) hingewiesen.
\end{abstract}

\section{BIBIONIDAE.}

Plecia tergorata Rond.

Plecia Wied.

Air Njuruk, Dempu, August.

\section{TIPULIDAE.}

Dicranomyia Steph.

Dicranomyia signata n. sp. Taf. II, Fig. 1.

Piek von Korintji, August, 1 o'.

Kopf samt Fühleru und Tastern schwarzbrain; das 1ste Geisselglied heller, die weiteren Glieder kurz eiförmig, kurz beborstet; Augen auf der Stirne zusammenstossend; Prothorax relativ lang, halsförmig. Thorax dunkelbraun mit linienförmiger weisslicher Zeichnung; es zeigt sich eine breite dunkelbraune Mittelstrieme, durch 2 weissliche Linien eingefasst, während die Seiten einige durch weisse Linien getrennte dunkle Flecken aufweisen; hinter der Quernaht eine weisslich schimmernde Mittelstrieme, welche sich auch auf das Schildchen 
fortsetzt. Brustseiten weisslich mit dunkleren Fleckchen. Hinterleib dunkelbraun, die Einschnitte wenigstens unten heller schillernd. Flügel glashell mit ausgedehnter dunkler Zeichnung; Vorderrand mit 5 grossen Flecken, z. T. mit helleren Kernen, Hinterrand mit 3 Flecken vor $\mathrm{Cu}_{2}$. Hintere Querader eine Strecke weit vor der Discoidalzelle. Schwinger gelb mit schwarzem Knopf. Beine alle abgebrochen.

Körperlänge ca. $6 \mathrm{~mm}$; Flügellänge $9 \mathrm{~mm}$.

\section{Limnobia Meig.}

Limnobia stigmosa n. sp. Taf. II, Fig. 2.

Suban Ajam, Juli.

Stirne mattgelb, Hinterkopf oben glänzend braun, der untere Teil des Kopfes gelb, Rostrum und Taster schwarz. Fühler relativ kurz, die beiden Wurzelglieder gelb, die Geissel schwarz, ziemlich lang beborstet. Thorax glänzend gelb, mit 4 glänzend schwarzen Striemen, von welchen die mittleren hinten die Quernaht nicht erreichen, die seitlichen vorn abgebrochen sind; hinten sind diese, auf den Schwielen hinter der Quernaht, verbreitert, hinten eingekerbt oder hier in 2 Fleckchen geteilt. Brustseiten, Schildchen und Hinterrücken ganz glänzend gelb. Hinterleib gelb, mit grossen, drejeckigen bis trapezförmigen schwärzlichen Mittelflecken, welche bisweilen nur den' Seitenrand freilassen; die 2 vorderen Segmente bisweilen ganz gelb oder nur hinten verdunkelt. Flügel etwas braungelblich, das Geäder sehr stark, das Stigma sehr gross, länglich, tiefschwarz, den Vorderrand und $R_{z+z}$ breit berührend, die queraderähnliche Wurzel von $\mathrm{R}_{3+4}$ schwarz gesäumt, dieser Saum das Stigma berührend, ferner ein schwarzer Flecken auf der Biegung an der Wurzel des sector radii; die übrigen Adern, wenigstens in der distalen Flügelhälfte, sehr schmal dunkel gesäumt. Hintere Querader an der Wurzel der Discoidalzelle. Beine relativ stark, schwarz, Hüften, Schenkelwurzel und ein breiter Ring vor der Schenkelspitze gelb.

Körperlänge $7-10 \mathrm{~mm}$, Flügellänge $12-15 \mathrm{~mm}$.

Limnobia annulifemur de Meij.

Suban Ajam, Juli.

\section{Libnotes Westw.}

Libnotes tripunctata n. sp. Taf. II, Fig. 3.

Suban Ajam, Juli, 1 \&.

Kopf 'samt Fühlern und Tastern mattschwarz, auch die sehr schmale Stirne von dieser Farbe. Thorax hellgelb, mit dunkler, durch eine feine Linie geteilter Mittelstrieme, welche sich fast bis zur Quernaht erstreckt. Hinterleib gelb, mit dunklen Vorderrandsäumen. Legeröhre kurz, die oberen Klappen nicht länger als die unteren, nur etwas gebogen. Flügel glashell, mit 3 dunkleren Stellen, an der Wurzel des sector radii zwischen Sc und $\mathrm{R}_{1}$, weiterhin am. Vorderrande an der Spitze von Se und $R_{1}$; Marginalquerader nur wenig länger als der Endabschnitt von $\mathrm{R}_{1}$. Hintere Querader etwas vor der Mitte der Discoidalzelle. Schwinger dunkel, nur die Basis des Stieles heller. Das einzig vorhandene Vorderbein braun.

Körperlänge ca. $7 \mathrm{~mm}$; Flügellänge $8 \mathrm{~mm}$.

Libnotes poeciloptera $0 . \mathrm{S}$.

Air Njuruk, Dempu, August, auch kleinere Exemplare von ca. $11 \mathrm{~mm}$ Flügellänge, Piek von Korintji, August.

$$
\text { Acyphona Ost. Sack. }
$$

Acyphona punctulata n. sp. Taf. II, Fig. 4.

Sungai Kumbang, September, 1 o.

Stirne gelblich, Fühler gelb, mit länglich ovalen Geisselgliedern. Rüssel und Tạster schwarz. Thorax mattgelblich, mit 2 genäherten dunklen Längslinien, welche sich, weiter 
von einander getrennt, auch hinter der Quernaht fortsetzen, auch am Seitenrande eine braune Längsstrieme; Schildchen gelblich. Behaarung des Thorax relativ lang, gelb. Brustseiten braun, weisslich bereift. Hinterleib dunkelbraun, gelb behaart; Legeröhre glänzend braungelb, obere Klappen dünn, etwas aufgebogen, so lang wie die unteren. Flügel glashell mit gelbem Geäder, die Queradern schmal schwarz gesäumt, die Längsadern abwechselnd mit dunklen und hellen Stellen, sodass der Flügel demjenigen gewisser Anophelinen ähnlich sieht. Discoidalzelle geschlossen, langgestreckt. Schwinger gelb. Schenkel dunkelbraun, an der Spitze und vor der Spitze mit gelbem Ring. Schienen und Tarsen gelb, letztere nur an der Spitze verdunkelt.

Körperlänge $5 \mathrm{~mm}$; Flügellänge $6 \mathrm{~mm}$.

Eine ähnliche Flügelzeichnung besitzt Erioptera punctipennis Brun. aus Vorderindien; sie hat eine offene Discoidalzelle und längere Analader.

\section{Gnophomyia Ost. Sack.}

Gnophomyia fascipennis n. sp. Taf. II, Fig. 5.

Muara Sako, September, 1 or.

Stirne dunkel rötlich braun, Fühlerwurzelglieder rötlich, die Geissel schwarzbraun, das $1^{\text {te }}$ Wurzelglied schmal, kurz cylindrisch, das $2^{\text {te }}$ geschwollen, rundlich, die Geisselglieder kurz, rundlich. Thorax und Hinterleib schwarz, der Thorax mässig glänzend; Flügel glashell mit 3 breiten schwarzen Querbinden, welche sich alle vom Vorder- bis zum Hinterrand erstrecken. Die mittlere lässt die Discoidalzelle und einen grossen Teil der darunter liegenden Zelle frei, doch sind die die Discoidalzelle distalwärts begrenzenden Queradern breit schwarz gesäumt. Die marginale Querader liegt vor der Wurzel der Radialgabel, die hintere Querader nur etwas jenseits der Discoidalzelle. Schwinger schwarzbraun. Beine desgleichen, die Schenkelspitzen nur wenig verdickt. Flügellänge $5 \mathrm{~mm}$.

Durch das Geäder schliesst sich diese Art den Gnophomyien an, andererseits zeigt sie auch grosse Ähnlichkeit mit Gn. ornatipennis de Meij., welche Alexander (Proc. U. S. Nation. Mus. Bd. 49, p. 16\%) in die Gattung Paratropesa zu stellen geneigt ist. Bei dieser Art ist $R_{2}$ viel steiler und liegt die marginale Querader an diesem Zinken der Radialgabel. Das Geäder der typischen Paratropesa singularis Schin. (Novara-Dipteren Taf. II, Fig. 2b) ist indessen doch wesentlich anders, obgleich auch hier die Radialgabel vorhanden ist, also $R_{2}$ und $\mathrm{R}_{3}$ gesondert zum Rande verlaufen, während sie bei den übrigen Antochinen bis zum Rande verschmolzen sind. Ich gestehe gerne, dass es gerade durch Paratropesa und Arten wie Gn. ornatipennis schwer hält eine Grenze zwischen den Gnophomyia-ähnlichen Eriopterinen und den Antochinen zn ziehen, möchte aber in der neuen Art eine Andeutung sehen, dass $G_{r}^{r} n$. ornatipennis doch besser in diese Gattung zu belassen ist. Osten Sackens Ansicht, nach welcher der queraderähnliche Aderzweig nicht eigentlich dem oberen Ast der Radialgabel $\left(R_{2}\right)$ homolog, sondern nur eine überzählige Querader sei, kann ich mich nicht anschliessen und halte seinen Versuch, in dieser Weise allen Antochinen den Besitz der Radialgabel abzusprechen, für verfehlt.

Gnophomyia ornatipennis de Meij. lag Riedel auch von Formosa vor (Entom. Mitt. II, 1913, p. 273).

\section{Mongoma Westw.}

Mongoma nigriceps $\mathrm{n}$. sp.

Suban Ajam, Juli, 1 o und ein Ex. mit abgebrochener Hinterleibsspitze.

Kopf mattschwarz, Augen auf der Stirne zusammenstossend. Fühler dunkelbraun, die beiden Wurzelglieder gelb, die Geisselglieder langgestreckt. Thorax ganz matt rotgelb. Hin-' terleib oben fast ganz schwarzbraun, mässig glänzend, die äusserste Wurzel und der Seitenrand schmal, hinten etwas breiter, gelb. Legeröhre glänzend schwarzbraun, die kleinen oberen 
Klappen aufgebogen. Flügel glashell, die Costalzelle gelblich, die kleine Subcostalzelle etwas dunkler. Aus der Discoidalzelle treten 4 Längsadern zum Aussenrand. Hintere Querader an oder etwas jenseits der Wurzel der Discoidalzelle. Schwinger schwarzbraun, der Stiel z. T. gelb. Beine braun, die Tarsen etwas mehr gelblichbraun.

Körperlänge $12 \mathrm{~mm}$; Flügellänge $10 \mathrm{~mm}$.

Die Art sieht $M$. cariniceps Enderl. offenbar sehr ähnlich, welche sich indessen durch hellere'Stirne, Fühler, Schwinger und Hinterleib unterscheidet: Auch zeigen sich einige Verschiedenheiten im Geäder, bei meiner Art ist $R_{2+3}$ am Flügelrande nicht S-förmig zurückgebogen und die unterste der die Discoidalzelle distal abschliessenden Queradern ist länger und gebogen. Obgleich Enderlein (Zool. Jahrb. Abt. System, Bd. 32, 1912, p. 61) nur das $\sigma^{7}$ beschreibt, Lalte ich meine Art doch nicht für das zugehörige $\%$, zumal Alexander (Proc. U. S. Nation. Mus. Bd. 49, 1916, p. 173), welcher die Art von Java erwähnt, angibt, dass das $ᄋ$ wie das $0^{T}$ gefärbt ist.

\section{Epiphragma Ost. Sack.}

Epiphragma Kerberti n. sp. Taf. II, Fig. 6.

Air Njuruk, Dempu, 1400 M., August; Suban Ajam, Juli.

Stirne braungelb, matt, zwischen den Augen ein grosser, dunklerer Mittelfleck, auch der hintere Teil des Kopfes oben dunkelbraun. Fühler dunkelbraun, das lte Geisselglied gelb, die Geissel weiterhin schwarz. Rostrum gelb, die Spitze und die Taster fast ganz schwarzbraun. Thorax grösstenteils matt dunkelbraun mit hellerer Zeichnung an den Nähten, vorn mit braungelber, durch eine dunkle Linie geteilter Mittelstrieme, welche sich in der Mitte der vorderen Thoraxpartie nach den Seiten umbiegt, median sich nur als feine Linie fortsetzt. Brustseiten weisslich mit dunkleren Fleckchen; Schildchen und Hinterrücken dunkelbraun, letzterer mit weisslicher Zeichnung. Hinterleib dunkelbraun, mit helleren Einschnitten und sehr schmalem, wenig deutlichem, hellerem Ring in der Mitte der Segmente. Flügel mit verwickelter Zeichnung von fast einfarbig dunkelbraunem Ton, die Randflecken an den Aderspitzen in der distalen Hinterrandshälfte mit grossem helleren Kern. Hüften weisslich mit dunkelbraunem Querring; Schenkel dunkelbraun, an der Spitze schmal etwas heller; Schienen und Tarsen gelb.

Körperlänge $10 \mathrm{~mm}$; Flügellänge $14 \mathrm{~mm}$.

Diese schöne Art widme ich gerne Herrn Dr. C. Kerbert, dem Direktor der Kgl. Zoolog. Gesellschaft "Natura Artis Magistra" zu seinem \%0ten Geburtstag.

Epiphragma insignis v. d. Wulp.

Air Njuruk, Dempu, August, 1.

Limnophila Macq.

Limnophila Palmeri Alex.

Sungai Kumbang, August, 1 . .

Teucholabis Ost. Sack.

Teucholabis plecioides de Meij.

Air Njuruk, Dempu, 1 \%.

Es ist heller als die javanischen Stücke, auch der Hinterleib ist grösstenteils gelbrot, desgleichen Schwinger und Hüften. In der Legeröhre ist keine Verschiedenheit zu bemerken. Auch die Flügel sind mehr gelblich und namentlich die Adern am Vorderrande heller.

\section{Eriocera Macq.}

Eriocera xanthopyga de Meij.

Air Njuruk, Dempu, 1 ơ, August. 
1. ㅇ von Muara Sako gehört wohl gleichfalls zu dieser Art, obgleich der Hinterleib ganz schwarzbraun ist.

Agastomyia gen. n. Taf. II, Fig. \%

Stirne mässig breit, wenig gewölbt; Untergesicht ziemlich kurz, fast flach, nicht rüsselartig verlängert. Fühler sehr lang, fadenförmig, die Wurzelglieder sehr kurz, gelb, die Geissel sehr lang, $15 \mathrm{~mm}$ lang, ihre 14 Glieder sehr stark verlängert, das letzte Glied am kürzesten, aber doch $6 \times$ so lang wie breit, alle stabförmig und lang abstehend behaart; die Haare dunkelbraun, mit glatter Oberfläche, die Einpflanzungsstellen als kleine Warzen vorspringend. Taster mässig lang, das letzte Glied nicht verlängert. Thorax von gewöhnlicher Bildung. Hinterleib relativ dünn, das Hypopyg etwas verbreitert, mit relativ kleiner Zange, Flügel mit in der Vorderrandshälfte stark reduziertem Geäder. Sc an der Spitze sowohl mit dem Vorderrand als mit $R$ verbunden, zwischen dieser und der Discoidalzelle gehen nur 2 Längsadern zum Flügelrande, welche durch eine Querader verbunden sind, und $\mathrm{m}$. Er. als $\mathrm{R}_{2+3}$, bzw. $R_{4+5}$ zu deuten sind, während $R_{1}$ fehlt. Discoidalzelle geschlossen, 3 Längsadern zum Flügelrand sendend; die untere ist $\mathrm{Cu}_{1}$ und berührt breit diese Zelle. Die 2te Analader (Axillaris) ganz fehlend. Beine von gewöhnlicher Bildung.

Die Gattung gehört zu den Cylindrotominae. Schon durch die eigentümlichen Fühler steht sie der Gattung Stibadocera Enderlein (Zool. Jahrb. Bd. 32, 1912, p. 83) sehr nahe, von welcher sie besonders durch das Flügelgeäder verschieden ist. Bei Stibadocera ist Sc. an der Spitze nicht mit der Costa verbunden, $R_{1}$ ist als kurzes, queraderähnliches Ästchen vorhanden und die Querader zwischen $R_{2+3}$ und $R_{4+5}$ liegt viel weiter vom Flügelrande entfernt. Eine $2^{\text {te }}$ Stibadocera beschreibt Alexander von Java (St. metallica; Proc. U. S. Nation. Mus., Bd. 49, p. 178) und weist gleichzeitig darauf hin, dass auch Cylindrotoma quadricellula Brun. aus Vorder-Indien hieher gehört.

Agastomyia albitarsis n. sp. Taf. II, Fig. \%.

Sungai Kumbang, September, $1 \sigma^{7}$.

Kopf blassgelb, glänzend, auch die Wurzelglieder der Fühler von dieser Farbe, die Geissel schwarzbraun, desgleichen die Taster. Thorax oben dunkelbraun, stark glänzend, Schildchen und Hinterrücken etwas heller, Brustseiten glänzend braungelb. Hinterleib dunkelbraun, am $2^{\text {ten }}$ und $3^{\text {ten }}$ Ring mit schmalem, gelbem Ring vor der Spitze, 4ter-6ter mit schmalem, gelbem Hinterrandsaum, der Bauch heller. Hypopyg dunkelbraun, die schmal lamellenförmigen Endglieder gelblich. Flügel glashell; ganz ohne Stigma. Beine schwarzbraun, Hüften und Schenkelwurzel schmal gelb, Tarsen mit Ausnahme des schwarzbraunen Metatarsus (ob an allen Beinen ?) weiss (beim vorliegenden Exemplar ist nur ein Hinterbein vollständig vorhanden). Schwinger schwarzbraun.

Körperlänge ca. $8 \mathrm{~mm}$, Flügellänge $9 \mathrm{~mm}$.

$$
\text { Sphaerionotus gen. n. Taf. II, Fig. } 8 .
$$

Kopf klein, Stirne relativ kurz, gleichmässig gewölbt, zwischen den Augen überall gleichbreit. Untergesicht nur sehr wenig gewölbt, überall von gleicher Breite, ca. 1,5 mal so lang wie breit, vorn nicht rüsselartig verlängert, nur wenig den unteren Augenrand überragend. Fühler sehr kurz, Wurzelglieder und das 1 te Geisselglied gut entwickelt, die folgenden Glieder kurz und schmal, eine dünne, relativ lang beborstete Geissel bildend, etwa von der Höhe des Kopfes. Taster sehr kurz; die Gliederanzahl nicht deutlich erkennbar, die gleichfalls sehr kurze Unterlippe kaum überragend. Augen elliptisch. Thorax im vorderen Teil stark gedunsen und den Kopf überragend, hier aber nicht von konischer Gestalt, sondern breit gerundet. Hinterleib relativ lang und schmal, nach hinten etwas erweitert. Flügel bedeutend kürzer als der Hinterleib. Sc mit $R_{1}$ verbunden, von der Verbindung mit der Costa nur eine Spur am Vorderrande vorhanden. Discoidalzelle nicht gross, fünfeckig; aus ihr treten 3 Adern zum Flügelrand, von welchen beim vorliegenden Stück am rechten Flügel die obere ganz nahe 
dem Flügelrand kurzgegabelt ist. $\mathrm{Cu}_{1}$ berührt die Discoidalzelle nur in einem Punkt. Beine von gewöhnlicher Bildung; die Tarsen relativ dünn und lang, namentlich der Metatarsus.

Diese Gattung schliesst sich Stegasmonotus Enderlein (Zool. Jahr. Bd. 32, 1912, p. 11) nahe an. Die Angabe: "der Clypeus (Epistoma) ist doppelt so breit wie lang, an der Basis scharf abgesetzt und trägt in der Mitte des Vorderrandes einen breiten abgestutzten Zapfen" trifft aber nicht zu, ferner hat hier die vordere Thoraxpartie eine fast kegliche Gestalt und wird auch nicht erwähnt, dass die Taster rudimentär sind. Bei der Artbeschreibung findet sich nur die Angabe: "Palpen grünlich, Endglied grauschwarz”.

Sphaerionotus curtipennis n. sp. Taf. II, Fig 8.

Muara Sako, October, $10^{7}$.

Kopf mattgelb, auch die Wurzelglieder der Fühler von dieser Farbe, die Geissel schwarzbraun. Taster und Labellen dunkelbraun. Thorax von glänzend gelbbrauner Grundfarbe mit 3 glänzend kaffeebraunen. Striemen, von welchen die breite mittlere den Prothorax vorn erreicht; alle drei überschreiten hinten die Quernaht; das kleine, gewölbte Schildchen gelb; desgleichen der Hinterrücken, die Brustseiten : glänzend dunkelbraun. Die 5 erstern Hinterleibsringe matt braungelb, der Seitenrand sehr schmal schwarzbraun, der 6 te matt dunkel-

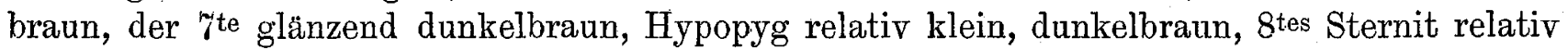
gross, hinten fast gerade abgeschnitten, 8tes Tergit kürzer, gerade abgeschnitten, 9tes Tergit kurz, gerundet, glänzend schwarzbraun, seitliche Anhänge von länglich dreieckiger Gestalt. Flügel gebräunt. Schwinger dunkelbraun, der Stiel nur an der Wurzel heller. Beine schwarzbraun, die Schenkel grösstenteils braungelb mit verdunkelter Spitze.

Körperlänge $17 \mathrm{~mm}$; Flügellänge $12 \mathrm{~mm}$.

\section{Pachyrrhina Macq.}

Pachyrrhina nigrithorax n. sp.

Air Njuruk, Dempu, 1400 M, August, 1: .

Stirne ganz matt schwefelgelb, sanft gewölbt; Rostrum schwarzbraun, Taster schwarz; Fühler tiefgelb, in der Endhälfte der Geissel die Glieder zunächst nur an der Basis, weiterhin ganz dunkelbraun; die Glieder sind an der Basis nicht verbreitert. Thorax ganz mattschwarz, also auch Schildchen, Hinterrücken und Brustseiten. Hinterleib in der Wurzelhälfte, bis zum Hinterrand des $4^{\text {ten }}$ Segmentes, gelb; oberseits sind indessen der $2^{\text {te }}-4^{\text {te }}$ Ring bis auf den Seitenrand stark gebräunt und ziemlich glänzend; כ̈ter-Yter Ring mattschwarz. Legeröhre glänzend schwarzbraun, oben an der Wurzel gelb, die oberen Klappen gerade, an der Spitze abgerundet. Flügel etwas bräunlich, die Costalzelle gelb, das Stigma braun, nicht besonders dunkel. Schwinger gelb. Hüften und Trochanteren. schwarz, Schenkel gelb, an der Spitze schmal schwarz, Schienen braun mit dunklerer Spitze, Tarsen schwarzbraun.

Körperlänge $14 \mathrm{~mm}$; Flügellänge $13 \mathrm{~mm}$.

Wegen des ganz mattschwarzen Thorax und der Färbung überhaupt erinnert diese Art stark an gewisse Eriocera-Arten.

Pachyrrhina familiaris 0 . S.

Air Njuruk, Dempu, 1 ᄋ.

\section{Tipula L.}

Tipula pseudofulvipennis n. sp.

Piek von Korintji, August.

In Studien über südostasiatische Dipteren V (Tijdschr. v. Entom., Bd. 54, 1911, p. 71 ) erwähnte ich ein o einer javanischen Tipula, welches unserer T. fulvipennis de G. sehr åhnlich sieht. Jetzt liegen mir aus Sumatra $1 \sigma^{7}$ und 1 o dieser selben Art vor, sodass auch die Genitalorgane zur Entscheidung über die Zusammengehörigkeit herbeigezogen werden können. 
Es ergibt sich anch in diesen eine grosse Übereinstimmung. Dennoch sind einige Unterschiede vorhanden, weshalb ich auch wegen der weit getrennten Fundorte sie als specifisch verschieden aufführe. Im übrigen genügt es auf die Differenzen hinzuweisen.

'Der T.' fulvipennis de G. äusserst ähnlich, die Zeichung in der distalen Hälfte der hinteren Basalzelle etwas mehr fleckig. Geisselglieder der Fühler dunkler, ganz schwarzbraun oder nur an der Spitze etwas heller. Thoraxstriemen wie bei fulvipennis verschieden deutlich, bisweilen sind deren 4 deutlich erkennbar, von welchen die 2 mittleren durch eine schmale helle Linie getrennt sind. Brustseiten auch bei dem sonst dunklen Männchen braungelb, mässig glänzend. Hypopyg von demselben Bau wie bei fulvipennis, die weisslichen appendices superae schmäler lamellenförmig; pars tertia der appendix intermedia derjenigen von fulvipennis ähnlich, braungelb, glänzend, die Endlappen der lamella terminalis infera schmäler und dunkler, und ohne die dichte und lange goldgelbe Behaarung, welche diesen Teil bei fulvipennis kennzeichnet.

Tipula praepotens Wied.

Rimbo Pengadang, Juni, 1 \%.

Tipula umbrina Wied.

Palupuh, Mai. Sungai Kumbang, 1 ․

\section{STRATIOMYIDAE.}

\section{Rosapha Walk.}

Rosapha variegata n. sp. Taf. II, Fig. 9.

Piek von Korintji, August, 1 o.

\$. Stirne glänzend schwarz. Stirnbreite fast wie bei habilis, der Schläfenrand etwas stärker entwickelt; Fühler gelbrot mit schwarzem Griffel; Untergesicht glänzend schwarz, am Augenrande schmal weiss bestäubt, Rüssel gelb, vorn dunkler. Thorax und Schildchen schwarz, punktiert, mässig glänzend; die 4 Dornen des Schildchens fast ganz gelb, die kurze Behaarung gelb. Hinterleib wie der Thorax. Flügel glashell, mit schwarzer Zeichnung: das Stigma und ein Wisch unter demselben, welcher unten schmal mit der breiten Verdunkelung der F'lügelspitze zusammenhängt, schwarzbraun; diese erstreckt sich bis zur Analzelle, hat jedoch

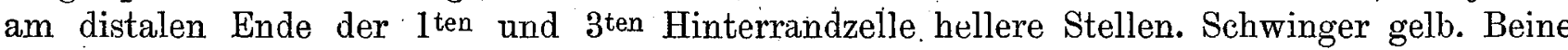
gelb. Vordertarsen ganz, von den hinteren Tarsen nur die Spitze, dunkelbraun; Metatarsus und das folgende Glied der Hintertarsen weissgelb. Körper- und Flügellänge $6 \mathrm{~mm}$.

Von bilineata unterschieden durch breitere Stirn, stärker gefärbte Flügel, von habilis durch die fast ganz glashelle vordere Radialzelle, von beiden durch ganz dunklen Thorax. Für diese beiden Arten vergleiche man Kertész, Ann. Mus. Nation. Hung. VII, 1909, p. 3\%6. Bezüglich der von mir in Stud. X, Tijdschr. v. Entom. LVIII, 1915, p. ; $_{2}$ beschriebenen $R$. obscurata + möchte ich noch bemerken, dass bei diesem der Ocellenhöcker auf der Stirnmitte, also vom hinteren Stixnrand beträchtlich entfernt liegt.

\section{Ceratothyrea de Meij.}

Ceratothyrea nigrifemur de Meij.

Air Njuruk, Dempu, August.

$$
\text { Cyphomyia Wied. }
$$

Cyphomyia obscuripalpis n. sp.

Air Njuruk, Derpu, $1400 \mathrm{M}$, August, 1 .

$\because \therefore$. Kopf gelb, nur der Ocellenpunkt schwarz. Stirne gleichmässig gewölbt, weder mit Höckern am Augenrande über den Fühlern nọch mit der Spur eines medianen Kammes. 
Unmittelbar über den Fühlérn eine seichte Querfurche. Fühler ganz schwarz, die Basalglieder kurz schwarz behaart. Untergesicht gelb behaart. Rüssel und Taster schwarz. Schläfenrand sehr stark entwickelt. Thorax schwarz, in der vorderen Hälfte median mit einer Strieme von gelbem Toment, an der Quernaht und hinten mit sehr kurzem, zerstreutem weissen Toment; Brustseiten dichter weiss tomentiert. Schildchen schwarz, die Dornen gleichfalls, mit kurzer, braungelber Spitze, nicht einwärts gekrümmt. Hinterleib stahlblau, namentlich hinten mit zerstreutem, sehr kurzem, weissern Tomente. Flügel schwarzbraun, an der Wurzel hell. Schwinger gelb. Beine schwarz, nur an den Mittelbeinen der Metatarsus braungelb. Körper- und Flügellänge ca. $9 \mathrm{~mm}$.

Die von Kertész (Ann. Mus. Nation. Hungar. XII. 1914, p. 505) gegebene Bestimmungstabelle führt auf curvispina Enderl., welcher sich durch gelbe Vorder- und Mitteltarsen unterscheidet, ferner durch die stark einwärts gekrümmten Dornen und durch gelbe Taster. C. flaviceps. Walk. hat wahrscheinlich ganz schwarze Beine, nach Kertész ist bei ihr die Spur eines Stirnkammes deutlich sichtbar.

Cyphomyia nigripes n. sp.

Air Njuruk, Dempu, 1400 M, August, 1 q.

․ Kopf tiefgelb, Ocellenhöcker dunkelbraun, Stirne in der Mitte vertieft, mit hohem Kamme in dieser Vertiefung. Schläfengegend stark entwickelt; Fühler schwarz. Untergesicht gelb behaart. Rüssel braun, Taster'schwarz. Thorax dunkel stahlblau mit ziemlich langer und dichter schwarzer Behaarung, mit wenig deutlicher, nur in der vorderen Hälfte erkennbarer Mittelstrieme von gelbem Tomente, hinten mit zerstreutem weissen Tomente; Schildchen und Dornen von derselben Farbe wie der Thorax, letztere wenig gebogen, weiss behaart. Brustseiten mit weissem Tomente. Hinterleib dunkel purpurn, grob gekörnelt, namentlich in der hinteren Hälfte ziemlich lang behaart. Flügel rauchbraun, nur die äusserste Wurzel glashell, das Stigma schwarzbraun. Schwinger weiss. Beine ganz schwarz. Körper- und Flägellänge $10 \mathrm{~mm}$.

Diese Art ist $C$. orientalis Kertész (Ann. Mus. Nation. Hungar. XII, 1914, p. 505) sehr ähnlich; diese unterscheidet sich durch die deutlich gelbe Spitze der Vorderschenkel und den gelben Vordermetatarsus, deutlichere Thoraxmittelstrieme, schwarzbehaarte Dornen, metallisch blaue Hinterleibsfarbe, schneeweisses Toment am Untergesicht.

\section{Ephippiomyia Bezzi.}

Ephippiomyia bilineata F.

Air Njuruk, Dempu, August.

\section{Ptecticus Löw.}

Ptecticus aeneithorax n. sp.

Suban Ajam, Juli.

Hintere Stirnpartie des $\sigma^{7}$ glänzend schwarz, die vordere weiss. Fühler gelb; Untergesicht braun; Rüssel und Taster gelb. Thoraxrücken glänzend. schwarzgrün. Schulterbeulen, Postalarcallus und Schildchen gelb. Brustseiten gelb; Mesopleurum schwarz; nur an den Rändern schmal gelb. Sternopleurum unten schwarz, Hypopleurum mit schwarzem Flecken. Hinterrücken schwarz. Hinterleib gelb, vom $2^{\text {ten }}$ Ringe an mit breiter schwarzer Querbinde. Hypopyg schwarz, glänzend, mit gelben Anhängen. Flügel gelblich, an der Spitze mehr bräunlich tingiert. Schwinger bräunlich. Beine gelb, die vorderen Tarsen schwarzbraun. Hinterschenkel unten in der Wurzelhälfte schwarz, was sich in der Mitte des Schenkels nach oben ausdehnt und dort einen Querring bildet. Hinterschienen schwarz; Hintertarsen weiss, an der Unterseite gelb. Körperlänge $14 \mathrm{~mm}$; Flügellänge $13 \mathrm{~mm}$.

Bein $Q$. ist die Stirne im grösseren hinteren Teile glänzend schwarz, vorne weiss. Unter den ähnlichen Arten unterscheidet sich longipennis durch die dunkle Flügelspitze, ganz gelbe 
Hinterschenkel, latifascıa durch braunen Thoraxrücken und Schildchen, schwarzen hinteren Metatarsus, sich weiter nach der Spitze hin erstreckende Schwärzung am Hinterschenkel.

Ptecticus tricolor v. d. Wulp.

Suban Ajam, Juli, 1 or.

Die Fühler sind grösstenteils schwarzbraun, nur an der äussersten Spitze et was heller braun.

\section{Microchrysa Löw.}

Microchrysa fuscistigma de Meij.

Air Njuruk, Dempu, August.

Sargus mactans Walk.

Sargus F.

Fort de Kock, November; Rimbo Pengadang, Juni.

\section{Campeprosopa Macq.}

Campeprosopa flavipes $\mathrm{Mg}$.

Sungai Kumbang, September.

Die Exemplare sind etwas dunkler als die meisten javanischen, die Mittel- und Hinterschenkel sind an der äussersten Wurzel schwarz, die Hintertarsen schwarz oder weiss; mit dem Geschlecht steht dies nicht in Beziehung. Der Hinterrand des Schildchens und die Dornen sind braungelb bis blauschwarz.

\section{Solva Walk.}

Solva tinctipes n. sp.

Suban Ajam, Juli; Piek von Korintji, September.

Stirne schwarz, gelb behaart; Fühler schwarz, die Basis an der Innenseite gelb; Untergesicht schwarz, Rüssel bräunlich, Taster gelbweiss. Thoraxrücken schwarz, kurz gelb behaart; Schulterbeulen, Streifen am Thorax-Seitenrande und Schildchen gelb, Seitenränder des letzteren schwarz, Brustseiten schwarz. Hinterleib schwarz mit schmalen gelben Einschnitten. Flügel etwas gebräunt, die untere Ader aus der Discoidalzelle erreicht den Hinterrand nicht ganz; $\mathrm{Cu}_{1}$ die Discoidalzelle nur in einem Punkt berührend. Schwinger gelb. Vorderhüften schwarz; Mittelhưften an der Basis schwarz, weiterhin gelb mit einem schwarzen Fleckchen vor der Spitze; Hinterhüften schwarz mit schmaler gelber Spitze. Vorderschenkel gelb, beiderseits mit schwarzer Längsstrieme, welche namentlich an der Aussenseite der Vorderschenkel sehr breit ist. Hinterschenkel unten und in der Wurzelhälfte schwarz, auch schmal an der Spitze, der übrige Teil gelb; Vorderschienen gelb, aussen mit dunklem Längsstrich, die hinteren, auch alle Tarsen, schwarzbraun. Hinterschienen aussen in der Wurzelhälfte mit weissem Längsstrich. Körper- und Flügellänge $8 \mathrm{~mm}$.

Diese Art sieht $S$. calopodata nach meiner Auffassung sehr ähnlich, welche sich aber durch ganz schwarze Hüften, nur am Unterrande schwarze Hinterschenkel, mehr glashelle Flügel u.s. w. unterscheidet. Auch meine completa hat ganz schwarze Hüften. Beim Exemplar von Korintji ist der Hintermetatarsus fast ganz weiss, die schwarzen Längsstriemen an den Beinen sind weniger entwickelt.

\section{Ceratosolva de Meij.}

Ceratosolva fascipennis n. sp.

Suban Ajam, Juli.

Stirne schwarz, vorn und vor dem Ocellenhöcker nit. weisser Behaarung. Fühler schwarzbraun, das $3^{\text {te }}$ und der Anfang des $4^{\text {ten }}$ Gliedes an der Innenseite weisslich gelb. Untergesicht schwarzgrau, Rüssel gelb, Taster weiss. Thorax schwarz, mit zerstreutem, sehr kurzem 
weissen Tomente. Schulterbeulen gelbweiss; Schildchen und Brustseiten schwarz. Hinterleib schwarz, am Seitenrande mit wenig auffälligen grauweiss behaarten Seitenflecken. Flügel etwas gebräunt, vor der Spitze mit breiter schwarzbrauner Binde, welche den. Hinterrand nicht erreicht. Die untere der 2 Adern aus der Discoidalzelle erreicht den Hinterrand nicht ganz; $\mathrm{Cu}_{1}$ die Discoidalzelle nur in einem Punkt berührend. Schwinger gelb. Beine, auch die Hüften, gelb, an den vorderen Beinen die Tarsen gebräunt, an den Hinterbeinen Hüften und Schenkel gelbrot, die Kniee schwarz, die Schienen in der Wurzelhälfte weiss, weiterhin schwarz, die 2 ersten Tarsenglieder weiss, die 2 letzten Glieder schwarz. Körperlänge $9 \mathrm{~mm}$; Flügellänge $8 \mathrm{~mm}$.

Ceratosolva cylindricornis de Meij.

Rimbo Pengadang, Juni; Muara Sako, October; Suban Ajam, Juli.

\section{LEPTIDAE.}

Chrysopilus Mạcq.

Chrysopilus egregius n. sp.

Air Njuruk, Dempu, $1400 \mathrm{M}$, August, $1 \sigma^{7}$.

$\sigma^{7}$. Augen breit zusammenstossend, das Stirndreieck dunkelgrau, über der Fühlerwurzel schmal weiss bestäubt. Fühler schwarzbraun. Untergesicht stark gewölbt, gelb; Rüssel-gelb; Taster braun. Thoraxrücken dunkelbraun, mit dichtem grünen Tomente, welches nur im Mittelfelde fehlt; Brustseiten braungelb. Schildchen dicht grün tomentiert. Hinterleib in der Wurzelhälfte braun, in der Endhälfte dunkler, schwarzbraun, namentlich hier mit zerstreutem weisslichen Tomente; Bauch an der Wurzel gelb, weiterhin schwarz. Flügel mit ausgedehnter verwaschener Bräunung der Spitzenhälfte und am Hinterrand; Stigma länglich schwarzbraun; der Vorderrandsteil in dieser Gegend' gelbbräunlich; Schwinger dunkelbraun. Hüften und Schenkel gelb, Schienen braun, Tarsen schwarzbraun. Flügel- und Körperlänge $10 \mathrm{~mm}$.

Chrysopilus obscuratus de Meij.

Sungai Kumbang, September; Suban Ajam, Juli.

Chrysopilus ungaranensis de Meij.

Sungai Kumbang, September; Air Njuruk, Dempu, August; Suban Ajam, Juli.

Chrysopilus lupinus 0. S. Taf. II, Fig. 10.

Suban Ajam, Juli; Air Njuruk, Dempu, 1400 M, August; Sungai Kumbang, September.

$\sigma^{7}$. Augen auch bei den Männchen schmal getrennt. Stirne tiefschwarz, hinten vor dem Ocellenhöcker weissgrau. Wurzelglieder der Fühler gelb, das 3té Glied schwarzbraun; hinter der Fühlerwurzel ist die Stirne schmal weiss bestäubt. Das sehr stark gewölbte Untergesicht wachsgelb. Rüssel gelb, Taster schwarz. Thorax oben matt braungelb, hinten mit zerstreutem grünlichen Tomente, Schildchen von derselben Farbe, Brustseiten gelb. Hinterleib braungelb mit schwach verdunkelten Einschnitten. Flügel gelb mit dunkler Zeichnung; die Spitze dunkel gesäumt; vom Stigma geht eine dunkle Binde längs dem unteren Zinken der Ràdialgabel bis zu diesem Saum, welcher durch eine ebensolche schmälere mit der Spitze der Mediastinalis verbunden ist; ausserdem ein paar Längsadersäume in der analen Region. Schwinger braun. Hüften und Schenkel gelb, Schienen und Tarsen schwarzbraun. Körperund Flügellänge $10 \mathrm{~mm}$. Beim $ᄋ$ ist die Stirn breiter, schwarzbraun seidenartig glänzend, gleichfalls vorn schmal weiss.

Bei den Exemplaren von Sungai Kumbang, welche auch etwas kleiner sind, ist die Flügelzeichnung weniger vollständig, beim einen am Flügelrand mehr in Flecke aufgelöst, beim $2^{\text {ten }}$, ist der 8tigmaflecken nicht mit dem distalen Saum verbunden, während die $2^{\text {tè }}$. Binde nur am untern Flügelrande entwickelt ist. Namentlich 'das letzte schliesst sich der 
von Osten Sacken beschriebenen Type in der Flügelfärbung an; es ist gleichfalls ein 오. In Abweichung mit seiner Beschreibung sind die Taster bei dieser Art gewöhnlich. schwarz und die Brustseiten nicht matt.

\section{Leptis F.}

Leptis incurvatus de Meij.

Suban Ajam, Juli; Sungai Kumbang, September.

Die Exemplare haben die streifenförmige Flügelfärbung von $L$. arcuatus, sodass im Zusammenhang mit der in meinen Studien über südostasiatische Dipteren VIII, p. 25 angegebenen eigentümlichen Durchflechtung der Merkmale es sich hier wohl um eine, in mehreren Hinsichten variable Art handelt.

\section{TABANIDAE.}

Haematopota Mg.

Haematopota lunulata Macq.

Piek von Korintji, August.

Haematopota irrorata Macq.

Air-Gaung Ketjil, August; Piek von Korintji, August; Suban Ajam, Juli.

BOMBYLIIDAE:

Hyperalonia Rond.

Hyperalonia chrysolampis Jaenn.

Air Njuruk, Dempu, August.

\section{ASILIDAE.}

Laphria Mg.

Maira flavifacies Macq.

Suban Ajam, Juli; Sungai Kumbang; Air Njuruk, Dempu, August.

\section{Maira Schin.}

Maira gracilicornis de Meij.

Suban Ajam, Juli; Air Njuruk, Dempu, August.

Die Behaarung des Untergesichtes ist weiss oder gelb.

Anoplothyrea de Meij.

Anoplothyrea javana de Meij

Muara Sako, October; Sungai Kumbang, September; Suban Ajam, Juli; Air Njuruk, Dempu, August.

Die sumatranischen Exemplare sind im allgemeinen etwas dunkler als die javanischen, die Borsten am Thoraxrande sind schwarz, auch zeigt die sehr kurze Thoraxbehaarung weniger gelben Schimmer.

\section{Promachus Löw.}

Promachus inornatus v. d. Wulp.

Air Njuruk, Dempu, August.

Synolcus Löw.

Synolcus bengalensis Macq.

Muara Sako, October, 1 o. 
Ommatius Wied.

Ommatius dilatipennis v. d. Wulp.

Suban Ajam, Juli; Pasir Ganting, October.

Ommatius argentatus de Meij.

Suban Ajam, Juli.

Ommatius argyrochirus v. d. Wulp.

Suban Ajam, Juli; Air Njuruk, Dempu, August (die gewöhnlich silbernfarbige Beinbehaarung ist bei diesem Stücke goldgelb); Muara Sako, October; Sungai Kumbang, September.

\section{EMPIDIDAE.}

Hybos Mg.

Hybos pilosipes $\mathrm{n}$. $\mathrm{sp}$.

Suban Ajam, Juli.

$\sigma^{7}$. Fühler schwarz, das 3te Glied eiförmig. Rüssel und Taster schwarz. Untergesicht weissgrau bestäubt. Thorax stark glänzend schwarz, nur hinten jederseits mit 1 Dorsocentralborste; Schildchen mit 2 schwarzen Borsten. Brustseiten schwarz, dünn grau bereift. Hinterleib schwarz, dünn braun bereift; Hypopyg mässig verdickt. Flügel mässig stark gebräunt, das lange Stigma dunkelbraun; Discoidalzelle so lang wie der letzte Abschnitt der $4^{\text {ten }}$ Längsader, die sie distal abschliessende Querader bedeutend kürzer als der letzte Abschnitt der $5^{\text {ten }}$ Längsader, $3^{\text {te }}$ und $4^{\text {te }}$ Längsader parallel. Schwinger gelb. Beine schwarz, lang schwarz behaart, die 2 ersten Glieder der Mittel- und Hinterbeine rotgelb, desgleichen breit die Kniee der Hinterbeine. Dornen an der Unterseite der Hinterschenkel kurz. Hinterschienen gerade.

Körper- und Flügellänge $6 \mathrm{~mm}$.

Diese Art sieht, auch in der Grösse, $H$. discoidalis de Meij. sehr ähnlich, diese hat aber eine längere Discoidalzelle und ganz schwarze Beine (nur die äusserste Basis der Hinterschienen ist etwas rötlich), welche auch wesentlich weniger behaart sind, auch sind die bei der vorliegenden Art nur kurz haarförmigen Praescutellarbrusten bei ihr viel deutlicher borstenartig. Durch die Beinfarbe zeigt die Art Ähnlichkeit mit geniculata v. d. W. nach meiner Auffassung, welche aber viel kleiner ist und glashelle Flügel besitzt mit schwachem Stigma und gelblicher Wurzel. In den Bemerkungen über diese Art (Studien VI, p. 323) ist Zeile 9. v. 0. Schienen statt Schenkel zu lesen.

Hybos longisetosus $\mathrm{n}$. sp.

Air Njuruk, Dempu, 1400 M; August, 1 ठ7.

$\sigma^{7}$. Fühler schwarz, das $3^{\text {te }}$ Glied eiförmig. Rüssel schwarz, Taster dunkelbraun. Thorax glänzend schwarz, namentlich in der hinteren Hälfte dünn weisslich bereift, hinten mit fast in einer Querreihe gestellten' gleichlangen Borsten. Schildchen schwarz, mit 2 wenig divergenten schwarzen Borsten. Brustseiten grauweiss bereift. Hinterleib schwarz, mit langer heller Behaarung. Hypopyg mässig verdickt. Flügel glashell, das Stigma gebräunt; Discoidalzelle so lang wie der letzte Abschnitt der 4ten Längsader, die sie distal abschliessende Querader viel kürzer. als der letzte Abschnitt'der 5ten Längsader, 3te und 4te Längsader parallel, 4te im letzten Abschnitt etwas geschwungen. Schwinger gelb, Beine schwarz, an den Vorderbeinen die äusserste Basis der Schienen und die beiden ersten Tarsenglieder mit Ausnahme ihrer Spitze, an den hinteren die Schienenwurzel in etwas grösserer Ausdehnung, überdies an den Mittelbeinen die 2 ersten Glieder, an den Hinterbeinen das 1ste Glied der Tarsen gelb. Die Mittelschienen tragen aussen und innen eine sehr lange Borste; an den Hinterschenkeln finden sich oben an der vom Körper abgewandten Seite 4 mässig starke Borsten; die Dornen an der Unterseite sind z. T. noch etwas länger als die Dicke des Schenkels beträgt. Hinterschienen gerade. Körper- und Flügellänge $3 \mathrm{~mm}$. 
Geniculata, der sie in der Grösse ähnlich sieht, unterscheidet sich durch glänzenderen Thoraxrücken, schwarze Vorderbeine, breit gelbe Kniee der Hinterbeine. Bezzis beide neue Arten von Formosa, major und tibialis, unterscheiden sich von den beiden hier aufgeführten Arten dụrch die schwarzen Beine, mit höchstens schmal gelben Knieen.

\section{DOLICHOPODIDAE.}

\section{Tachytrechus Walk.}

Tachytrechus argentatus n. sp., Taf. II, Fig. 11.

Piek von Korintji, August.

Stirne und Untergesicht silberweiss. Fühler schwarz, das 3te Glied kurz eiförmig. Wimpern am hinteren Augenrande schwarz. Thorax metallischgrün, dicht mit Silberschimmer übergossen, welcher nur ganz hinten und auf dem zweiborstigen Schildchen kaum vorhanden ist. Brustseiten grau, weiss bereift. Hinterleib ziemlich dunkel metallischgrün, gleichfalls intensiv silbern schimmernd, das Hypopyg sehr gross, schwarz, mit herzförmigen äusseren Anhängen. Flügel etwas gebräunt, im distalen Teil am Vorderrande mit einem verwaschenen dunkelbraunen Wisch; 3te und $4^{\text {te }}$ Längsader gebogen, stark convergierend; hintere Querader senkrecht und gerade; Schüppchen gelb, schwarz gewimpert; Schwinger gelb. Vorderhüften gelb, nur an der Basis graulich; hintere Hüften grau; Schenkel und Schienen gelb, die Hinterschenkel an der Spitze schmal schwarz. Vordertarsen schwarzbraun, an den vorderen der Metatarsus fast ganz gelb, überdies an den Vordertarsen das $5^{\text {te }}$ Glied verbreitert und weiss, das $4^{\text {te }}$ auch etwas breitgedrückt, aber schwarzbraun. Körper- und Flügellänge 4,5 mm.

Die sich auf die europäischen Arten beziehende Gattungscharakteristik kommt bei den malayischen einigermaassen ins Gedränge. Bei argentatus findet sich an der Spitze der Hinterschenkel nur eine Borste, das Untergesicht ist verlängert aber im unteren Teile nicht wesentlich verbreitert. Dagegen erreicht das Untergesicht bei meinem $T$. crassitarsis nicht eigentlich den, unteren Augenrand; die Beugung der $4^{\text {ten }}$ Längsader und die zu mehreren vorhandenen Borsten am Ende der Hinterschenkel sprechen aber für diese Gattung. Bei argentatus ist die $4^{\text {te }}$ Längsader gleichmässiger geschwungen, wie bei mehreren Gymnopternus-Arten.

\section{Argyra Macq.}

Argyra splendida n. sp.

Suban Ajam, Juli; Piek von Korintji, August; Sungai Kumbang, September; auch Tjibodas (Java), Februar.

$\sigma^{7}$. Stirne und Untergesicht silbernschimmernd. Fühler schwarz, das 3te Glied länglich eiförmig, die Borste etwas vor der Spitze eingefügt, das 1 te Fühlerglied oben beborstet. Thorax und Hinterleib dicht mit Silberschimmer übergossen, welcher nur am hintersten Teil des Thoraxrücken fehlt. Thorax grün, hinten und auf dem Schildchen mit bläulichen Reflexen. Hinterleib schwärzlich mit breiten, viereckigen Seitenflecken am $2^{\text {ten }}$ und $3^{\text {ten }}$ Ringe, die des 2 ten am längsten. Brustseiten und Hüften grau, weiss bereift. Schenkel gelb, alle an der Wurzel etwas, die Hinterschenkel auch an der Spitze verdunkelt; die Schenkel unten ziemlich lang weitläufig schwarz gewimpert; Schienen gelb, die Hinterschienen im Enddrittel schwarz; Vordertarsen gelbbraun, Hintertarsen schwarz. Flügel etwas bräunlich tingiert, namentlich im distalen Teile des Vorderrandes. 4te Längsader im Endabschnitt deutlich geschwungen; hintere Querader stark geschwungen, namentlich unten. Schüppchen breit schwarz gerandet, mit gelben Wimpern, Schwinger gelb. Körperlänge $8 \mathrm{~mm}$; Flügellänge $6 \mathrm{~mm}$.

Die Brăunung am Vorderrande ist sehr verschieden stark, bisweilen fast fehlend, bisweilen, namentlich bei dem Exemplar von Korintji, im distalen Teile sehr stark. Vom o. liegt nur ein Exemplar aus Suban Ajam vor; die Stirne ist dunkel metallischgrün, das Untergesicht weiss bestäubt; das 3te Fühlerglied kürzer eiförmig. Der, Thoraxrücken ist nur vorn weiss bestäubt, weiterhin metallischgrün mit 3 kupferrötlichen Längsstriemen. Hinterleib 
schwärzlich, an der Basis namentlich an den Seiten grünlich, nur von gewisser Seite etwas weisslich bereift erscheinend; Schenkel weniger und kürzer bewimpert als beim $\sigma^{7}$, an den vorderen Schenkeln nur nahe der Spitze. Flügel im distalen Teile des Vorderrandes mässig verdunkelt.

Von Argyra-Arten war bisher aus dem Gebiete nur erst sehr wenig bekannt. A. spinipes Dol. ist blau, mit rötlichen Fühlern und Beinen, Stirne und Untergesicht sind silbernschimmernd. Mir blieb sie bis jetzt unbekannt.

Psilopus vittatus Wied.

Psilopus Mg.

Muara Sako, October.

Psilopus Jacobsoni de Meij.

Suban Ajam, Juli; Air Njuruk Dempu, August.

Sympycnus apicalis de Meij.

Sympycnus Löw.

Suban Ajam, Juli, 1 o'.

Sympycnus brevinervis de Meij.

Suban Ajam, Juli, 1 ㅇ.

Wie in der Beschreibung der Type angegeben (Tijdschr. v. Entom. LIX, p. 248) ist die Fühlerwurzel schwarz, das 3te Glied gelblich. In der Tabelle auf p. 245, 246 ist bei №. 5 $3^{\text {tes }}$ Fühlerglied statt Fühlerwurzel zu lesen. - Das Schildchen ist ganz gelb; die hintere Querader etwas länger.

\section{SYRPHIDAE:}

Microdon novaeguineae de Meij.

Microdon Mg.

Air Njuruk, Dempu, August; Suban Ajam, Juli, auch $1 \sigma^{7}$; das Hypopyg ist gelbrot.

Die Art war bis jetzt nur von Neu-Guirea bekannt.

Callicera Panz.

Callicera sumatrensis n. sp.

Suban Ajam, Juli, 1 ㅇ.

Stirne glänzend schwarz, schwarz behaart, Fühler schwarz, die 2 ersten Glieder fast gleichlang, das 3te von doppelter Länge, mit kurzer Endborste. Untergesicht glänzend bläulich schwarz, an den Seiten breit weiss bestäubt, mit weisser Behaarung. Augen dicht braun behaart, Thorax glänzend schwarz, im Mittelfelde der vorderen Hälfte mit 2 dicht genäherten weisslichen Striemen. Die ziemlich lange Thoraxbehaarung ist vorne dunkelbraun, hinten schwarz, auf dem wenig glänzenden, schwarzbraunen Schildchen dunkel, an dessen Rande gelbweiss, an den Brustseiten gelblich. Hinterleib schwarz, vorn an den Seiten dicht gelblich behaart, oben kurz bräunlich, in der. Mitte schwärzlich behaart. Flügel etwas gebräunt, das Stigma blassbraun. Schwinger gelb. Hüften und Schenkel schwarz, weisslich behaart, die vorderen an der Spitze schmal gelbbraun; Schienen gelbbraun; Tarsen schwarzbraun, die Metatarsen der Mittelbeine gelbbraun.

Körperlänge $9 \mathrm{~mm}$; Flügellänge $9 \mathrm{~mm}$.

Xylota nigroaenescens Rond.

\section{Xylota $\mathrm{Mg}$.}

Air Njuruk, Dempu, 1400 M, Aagust.

Das Exemplar ist etwas grösser als Rondani angibt, die Körperlänge beträgt $13 \mathrm{~mm}$. 
Der Thorax . ist grünlich bronzefarben, das Abdomen zieht ins Purpurne. Untergesicht dicht weiss bestäubt. Thorax dicht aber sehr kurz gelblich behaart, auch das Schildchen. Die beiden in der vorderen Hälfte vorhandenen Längsstriemen gelblich. Die Vorderschienen sind an Basis und Spitze gelb, an den Vordertarsen die 3 ersten Glieder, nach Rondani nur die Mitte, wodurch die Identität nicht ganz feststeht. Der Hintertrochanter hat einen kurzen, aber starken Dorn. Hinterschenkel mässig verdickt, gelb behaart, unten aussen und innen mit einer Reihe von Börstchen. Hinterschienen stark gebogen. Die mattschwarze Zeichnung des Hinterleibs besteht am $2^{\text {ten }}$ Ringe aus einem mehr dreieckigen, am $3^{\text {ten }}$ aus einem halbkreisförmigen Hinterrandflecken.

In Records Indian Museum XI, 1915, p. 235 hat Brunetti eine X. bistriata aus Cochin beschrieben, welche in der Körperfärbung grosse Ähnlichkeit hat, die Beine sind aber viel heller.

\section{Arctophila Schin.}

Arctophila apiformis $\mathrm{n}$. sp.

Piek von Korintji, August, 1 o 1.

Kopf dicht gelblich bestäubt, Stirn dunkelbraun behaart. Fühler schwarzbraun mit ovalem letzten Gliede, am Untergesicht das Mittelfeld und eine Strieme vom Auge bis zum Mundrande dunkelbraun; die Behaarung von Untergesicht und Backen gelb. Thorax samt Schildchen dunkelbraun, mässig glänzend, die mässig lange Behaarung dunkelbraun; Brustseiten ins Graue ziehend, gelb behaart. Hinterleib braun, an der Wurzel etwas heller, obenauf sehr kurz behaart, vorn an den Seiten und an der hinteren Spitze länger rotgelb behạart. Flügel mässig gebräunt, in der Mitte mit vom Stigma bis zur Discoidalzelle sich erstreckender verwaschener Verdunkelung. Beine schwarzbraun, die Vorderschienen an der Wurzel, die mittleren samt Metatarsen braungelb. Die verdickten Hinterschenkel unten nahe der Spitze mit stumpfem Höcker.

Körperlänge $14 \mathrm{~mm}$; Flügellänge $12 \mathrm{~mm}$.

Diese wie eine Honigbiene aussehende Art ist kürzer behaart, als in dieser Gattung gebräuchlich, auch ist der Hinterleib etwas mehr gestreckt. Aus Asien war bis jetzt nur $A$. simplicipes Brunetti (Rec. Indian Mus. XI, 1915, p. 247) bekannt.

Milesia Latr.

Milesia minor n. sp.

Piek von Korintji, August, 1 o'; Gunung Singalang.

Kopf wachsgelb, gelb bestäubt. Fühler rotgelb. Untergesichtshöcker braun, Backen glän zend, hinten dunkelbraun; Hinterkopf unten gelb behaart. Thorax schwärzlich, von vorn gesehen mit 3 dunkelbraunen Längsstriemen; Schulterbeulen, das Vorderende der seitlichen Längsstriemen, eine linie an der Quernaht mattweiss bereift. Schildchen schwarzbraun; Brustseiten grösstenteils gelblich bereift, mit einigen braunen Stellen. Hinterleib dunkelbraun, schwach glänzend, die mittleren Ringe am Hinterrand schmal gelb, die kurze, anliegende Behaarung braun, gelblich schimmernd. Flügel schwach gebräunt, die Adern nahe der Flügelspitze breit verwaschen gesäumt, auch um die Adern in der Flügelmitte dunklere Säume. Schwinger gelb: Beine gelb, Schenkel gelbbraun, Vordertarsen dunkler braun. Hinterschenkel ohne Dorn.

Körperlänge $14 \mathrm{~mm}$; Flügellänge $12 \mathrm{~mm}$.

Das $\sigma^{7}$ vom G. Singalang unterscheidet sich namentlich durch die Hinterleibsfärbung; dieser ist in viel ausgedehnter Weise braungelb, sodass an den meisten Ringen nur die Vorderränder, in der Mitte etwas breiter, dunkelbraun sind. Bei dieser Art zeigt, ebenso wie bei simulans de Meij., die 1te Hinterrandzelle eine tiefe, Eristalis-ähnliche Einbuchtung, welche bei Milesia gewöhnlich nicht vorhanden ist. Wir haben es hier mit derselben Erscheinung zu tun' wie bei der nordamerikanischen Arctophila flagrans, bei welcher diese Einbuchtung gleichfälls als Ausnahme für diese Gattung vorhanden ist. $M$. simulans ist grösser, der Hin- 
terleib ist anders gefärbt, in der Mitte schwarz behaart, die Flügelspitze mehr verwaschen verdunkelt, ohne hellere Kerne in den Zellen, die kleine Querader ist in der unteren Hälfte stärker gebogen.

Milesia simulans de Meij.

Air Njuruk, Dempu, September; Suban Ajam, Juli.

Vorletzter Hinterleibsring mit grösserem, namentlich breiterem schwarzen Flecken als bei der Type; Stirne dunkler, der nicht gelb bestäubte Teil über der Fühlerwurzel schwarzbraun.

Milesia gigas Macq.

Air Njuruk, Dempu, August.

Megaspis Macq.

Megaspis zonalis $\mathbf{F}$.

Suban Ajam, Juli.

\section{Eristalis Latr.}

Eristalis niger Wied.

Air Njuruk, Dempu, August, 1 \%; Suban Ajam, $1 \sigma^{\top}$, mit am $2^{\text {ten }}$ Hinterleibringe grossen gelblichen Seitenflecken und mit glashellen Flügeln, nur das Stigma ist dunkel.

Volucella Geoffr.

Volucella trifasciata Wied.

Suban Ajam, Juli; Air Njuruk, Dempu, August.

\section{Graptomyza Wied.}

Graptomyza brevirostris Wied.

Suban Ajam, Juli.

Graptomyza Jacobsoni de Meij.

Suban Ajam, Juli.

Bei den 3 erbenteten Exemplaren ist der Hinterleib etwas dunkler als bei den javanischen, am $2^{\text {ten }}$ Ringe dehnt sich die schwarze Farbe weiter nach vorn aus und der 3te ist fast ganz schwarz, bis zum Vorderrande; der schwarze Seitenrandsaum ist nur durch einen schmalen gelben Längsstreifen von der ganz schwarzen Medianpartie getrennt; der 4te Ring ist ganz schwarz.

\section{Chilosia $\mathrm{Mg}$.}

Chilosia javanensis de Meij.

Suban Ajam, Juli.

\section{Syrphus F.}

Syrphus consequens Walk.

Suban Ajam, Juli.

Syrphus balteatus de G. Suban Ajam, Juli.

Syrphus aegrotus $\mathrm{F}$.

Suban Ajam, Juli.

Syrphus depressus de Meij.

Air Njuruk, Dempu, August, 1 ․

Es unterscheidet sich vom Männchen durch etwas stärkere Ausdehnung der schwarzen 
Hinterleibsfärbung, der Vorderrand des $3^{\text {tẹn }}$ und $4^{\text {ten }}$ Ringes ist schmal schwarz, am ăten Ringe findet sich eine $\Lambda$-förmige Figur.

Syrphus monticola de Meij.?

Piek von Korintji, August, $1 \sigma^{\top}$; an der äussersten Grenze des Pflanzenwuchses; wahrscheinlich mit monticola de Meij. von Java (Studien IX, p. 159) identisch, aber durch dunklere Beine verschieden, auch die vorderen Beine sind schwarz, nur die Kniee gelb.

Melanostoma Schin.

Melanostoma ceylonense de Meij.

Sungai Kumbang, August.

Baccha F.

Baccha Austeni de Meij.

Muara Sako, September; Sungai Kumbang, September.

Spheginobaccha de Meij.

Spheginobaccha macropoda Big.

Muara Sako, October.

\section{HELOMYZINAE.}

Helomyza Fall.

Helomyza grandis n. sp.

Sungai Kumbang, September.

$\sigma^{7}$. Kopf rotbraun, die kurzen Periorbiten und das Scheiteläreieck glänzend, vorne gelb, nach hinten in schwarzbraun verdunkelt; Fühler rotgelb, das 3te Glied länglich, am Rande und an der Spitze schmal schwarzbraun, die Borste beiderseits lang gefiedert, Untergesicht gelb, mit grossem, oben gezacktem dunklen Fleck, welcher den unteren Rand berührt, sodass man auch sagen könnte: dunkel, nur oben und am Seitenrande gelb, bisweilen ganz schwarz oder fast ganz gelb; Wangen dunkelbraun, glänzend, mit weisslichem Schimmer, auch der anschliessende vordere, unter dem Auge liegende Teil der Backen von dieser Farbe. Rüssel und Taster rotgelb. Augen breit oval. Thorax matt braungelb, oben mit 4 schwach verdunkelten Längsstriemen, die beiden äusseren sehr breit, die inneren, zwischen den beiden Dorsocentralborstenreihen liegenden, schmal, eine schmale gelbe Mittelstrieme zwischen sich übrig lassend, Schildchen gelbbraun, oben nur an den Seiten behaart, Brustseiten rotgelb, Mesopleura ganz nackt. Hinterleib schwarzbraun, mässig glänzend, unten zottig schwarz behaart. Flügel schwach gebräunt, an der Wurzel und am Vorderrande etwas stärker, an der Spitze der 2ten Längsader ein kleines dunkles Fleckchen. Beine rotgelb, der Vorderschenkel oben mit breitem schwarzbraunen Längsstrich, auch unten ein solcher Strich; Schienen und Tarsen schwarzbraun; Schwinger rotgelb. Schenkel alle stark, Vorderschenkel unten und Vorderschienen innen stark zottig behaart; Mittelschenkel vorne mit 4, hinten mit 1 Borste; an den hinteren Beinen die zottige Behaarung weniger lang. Körperlänge $10 \mathrm{~mm}$.

Das einzige $q$ zeigt ám Untergesicht die oben als typisch angegebene Färbung; es unterscheidet sich von dem ${ }^{*}$ haupsächlich durch die nicht verdickten und viel weniger behaarten Schenkel, ferner durch die intensivere Flügelfärbúng; die Spitze namentlich der 2ten, aber auch der $3^{\text {ten }}$ und $4^{\text {ten }}$ Längsader sind ziemlich lang dunkel gesäumt und am Flügelrande findet sich an dieser Stelle ein schmaler dunkler Saum, welcher beim o sehr schwach vorhanden ist; auch die Säumung der hinteren Querader ist breiter und duvkler. Am Hinterleib ist unten die Behaarung nur kurz. - Echte Helomyzen sind im orientalischen Gebiet spärlich. In van der Wulp's Catalogue findet sich eine Anzahl angegeben, aber nach Czerny's Untersuchungen sind dies alles entweder Sapromyzen, Trypetinen oder Drosophilinen (Czerny, 
Revision der Helomyziden, Wien. Entom. Zeitg. XXIII, 1904, p. 203, 204, 20\%). Nur von copiosa Walk. konnte dies, weil die Type nicht mehr vorhanden ist, nicht festgestellt werden.

\section{SCIOMYZINA E.}

Sepedon Latr.

Sepedon chalybeifrons de Meij.

Sungai Kumbang, September.

Sepedon violaceus Hend.

Sungai Kumbang, September.

Bis jetzt nur aus Calcutta und Formosa bekannt.

CALOBATINAE.

Calobata Mg.

Calobata prudens O. S.

Muara Sako, October.

Nerius F.

Nerius sumatrensis n. sp.

Suban Ajam, Juli, $2 \sigma^{\pi} \sigma^{\pi}$.

Stirne mattschwarz, die Periorbiten glänzend schwarz; nur die äusserste Vorderspitze der Stim mattgelb. Fühler schwarz, die weissliche Borste dünn, kurz behaart. Wangen und vorderer Teil der Backen gelb, auch Rüssel und Taster. Untergesicht schwach dunkler. Thorax ganz schwarz, mässig glänzend. Schulterbeulen am Oberrande braun; Schildchen mit 2 Borsten. Hinterleib schwarz, schwach glänzend, höchstens der lte Ring oben z.T. gelblich. Flügel grösstenteils mit gelblichem Tone, die Spitze breit schwarz; bisweilen ist noch deutlich zu erkennen, dass diese Bräunung aus Verschmelzung von Aderspitzensäumen entstanden ist. Schwinger gelb. Vorderhüften gelb, die hinteren schwarz; im übrigen sind die Beine fast ganz schwarzbraun, nur die Schenkel, namentlich die Vorderschenkel, an der Wurzel gelblich. Die Vorderbeine sind bei diesen Männchen wieder stark verlängert, die Schenkel unten an der vom Körper abgewandten Seite mit einer Reihe sehr kurzer, abstehender Dörnchen besetzt.

Körperlänge $5 \mathrm{~mm}$; Flügellänge $6 \mathrm{~mm}$.

Nerius tinctipennis n. s̀p. Taf. II, Fig. 12.

Suban Ajam, Juli, 1 q.

Stirne mattschwarz, vorn mit schmal dreieckigem mattroten Flecken, welcher sich bis zur Stirnmitte erstreckt. Periorbiten und Scheitel breit glanzend schwarz, auch die unmittelbar hinter der Fühlerwurzel liegenden Stirnteile bilden glånzend schwarze Schwielen. Fühler schwarz, das 3te Glied unten an der Wurzel mit rötlichem-Flecken; die Borste weiss, pubeszent. Wangen und Backen gelb, Untergesicht braun mit gelber Längsstrieme; Mundrand sehr schmal schwarz. Rüssel schwarz, oben an der Spitze z.T. gelb, Taster dunkelbraun. Thorax oben schwarzbraun, dünn weiss bereift, mit 2 wenig deutlichen, matt bräunlich weissen Längsstriemen, welche die Dorsocentralborsten enthalten. Brustseiten glänzend schwarz. Schildchen schwarz. Hinterleib ganz schwarz. Flügel gelb, mit ausgedehnter dunkler Zeichnung an der Flügelspitze, ausserdem die beiden Queradern dunkel gesäumt und ein dunkler Schatten über der hinteren in der $1^{\text {ten }}$ Hinterrandzelle. Schwinger gelb. Beine ganz schwarz, nur die Wurzel der Vorderschienen etwas heller. Körper- und Flügellänge $6 \mathrm{~mm}$.

Diese schöne Art geht etwas aus der Charakteristik der Gattung heraus, weil das Schildchen 4 stark entwickelte Borsten besitzt, während bei Nerius gewöhnlich deren nur 2 vorhanden sind. Dadurch stimmt die Art mit Telostylus überein, die Fühlerborste ist aber durchaus 
nicht rein apical, sondern deutlich eine kurze Strecke vor dem abgerundeten Ende des 3ten Fühlergliedes eingepflanzt. Die 3te Längsader ist im Spitzenteil nach unten geschwungen, die. $4^{\text {te }}$ gleichmässig sanft nach oben gebogen.

Nerius fuscus Wied.

Suban Ajam, Juli.

Nerius bilineatus de Meij.

Suban Ajam, Juli.

\section{Telostylus Big.}

T'elostylus trilineatus de Meij.

Air Njuruk, Dempu, August.

Telostylus babiensis de Meij. var.

In Tijdschr. v. Entom. LVIII, p. 37 beschrieb ich diese Art von der kleinen Insel Pulu Babi nahe Simalur; sie wäre besonders durch das ganz dunkle Schildchen kenntlich. Das vorliegende Stück, 1 q von Air Njuruk (Dempu), sieht dieser Art sehr ähnlich, ist aber noch dunkler, der ganze Thorax ist dunkel, oben schwarzgrau, die Brustseiten sind glänzend schwarz. Im übrigen gibt es auch unter dem Material von Pulu Babi Stücke mit fast ganz verdunkelten Brustseiten. Weil die Stirnfärbung bei allen diesen dieselbe ist wie bei meinem trilineatus liegt die Vermutung nahe, dass es sich hier im Ganzen um eine in der allgemeinen Farbe variable Art handle.

Telostylus niger Bezzi von den Philippinen ist offenbar eine sehr ähnliche dunkle Form, von welcher sich unser Exemplar durch median nicht nur über den Fühlern, sondern auch weiter nach hinten rote Stirne, ganz schwarze Legeröhre und durch schwarze Mittel- und Hinterschienen unterscheidet. Doch weist auch Bezzi an derselben Stelle (Philipp. Journ. Science. Vol. VIII, NNo. 4 D, 1913, p. 329) auf die mögliche grosse Variabilitat von T. binotatus Big. hin, was mit meiner obigen Vermutung stimmt.

\section{Gobrya Walk.}

Gobrya simulans de Meij.

Muara Sako, October.

Nothybus Rond.

Nothybus longithorax Rond.

Suban Ajam, Juli, 1 o.

Rondani's Beschreibung stimmt, nur bezieht sie sich offenbar auf das. $\sigma^{7}$. Bei dem vorliegenden $q$ sind vom Hinterleib der 1 te und $2^{\text {te }}$ Ring gelbrot, der 2te nach der Spitze hin dunkler, der $3^{\text {te }}$ und $4^{\text {te }}$ schwarzbraun, mässig glänzend, der $5^{\text {te }}$ mattschwarz, der 6 te und yte gelblich.

\section{SEPSINAE.}

Sepsis Fall.

Sepsis contracta Walk:

Sungai Kumbang, August, Kep. Alüang.

\section{DIOPSINAE.}

Diopsis L.

Diopsis indica Westw.

Muara Sako, October. 
Diopsis Dalmani Wied.

Muara Sako, September; Suban Ajam, Juli.

Teleopsis Rond.

Teleopsis Sykesi Westw.

Suban Ajam, Juli.

\section{ORTALINAE.}

Lule Speis.

Lule Speiseri de Meij.

Suban Ajam, Juli; Air Njuruk, Dempu, August.

Bei beiden Stücken ist der Thorax mehr bronzefarben als bei der kupfergrünen Type.

Xiria Walk.

Xiria antica Walk.

Muara Sako, October.

Xiria obliqua Ost. Sack.

Muara Sako, October; Air Njuruk, Dempu, August.

Loxoneur Macq:

Loxoneura decora $\mathrm{F}$.

Air Njuruk, Dempu, August, 1 \&.

Plagiostenopterina Hend.

Plagiostenopterina trifasciata $\mathrm{n} . \mathrm{sp}$.

Suban Ajam, Juli, 1 o'.

Stirne fast ganz mattschwarz, nur am Augenrande schmal dunkelbraun, mit weissem Schimmer; Scheiteldreieck und Scheitelplatten glänzend schwarz. Wurzelglieder der Fühler gelbrot, das 3te Glied schwarzgrau, Borste im Wurzeldrittel kurz behaart. Untergesicht und Backen braungelb. Wangen und die angrenzenden Fühlergruben weiss bestäubt. Taster braungelb; Rüssel schwarz. Hinterkopf schwarz.

Thorax erzgrün mit 3 breiten gelbbestäubten Längsstriemen, auf welchen die Behaarung gleichfalls gelb ist. Schildchen schwärzlich, gelb bereift. Brustseiten schwarz, hinterer Teil der Mesopleuren und die Sternopleuren weiss bereift mit weisser Behaarung. Hinterleib erzgrün, der $2^{\text {te }}$ Ring in der Mitte und namentlich hinten an den Seiten gelb mit gelber Behaarung; im übrigen die Behaarung dunkel. Flügel glashell, nur die schmale Subcostalzelle braun, die Flügelspitze am Rande kaum merkbar dunkler, Queradern nicht gesäumt, kleine Querader etwas schief gestellt, Schüppchen weiss, Schwinger weissgelb. Beine schwarz, die Vorderhüften und alle Trochanteren und der Hintermetatarsus bräungelb.

Körperlänge $8 \mathrm{~mm}$; Flügellänge $6 \mathrm{~mm}$.

Diese Art gehört in die Nähe von trivittata Walk., welche sich u. a. durch gelblich tingierte und mehr gezeichnete Flügel unterscheidet, auch sind die Schenkel rotgelb. Meine $P l$. vittigera aus Sumatra und Simalur ist gleichfalls sehr ähnlich.

Plagiostenopterina longivitta Walk.

Suban Ajam, Juli, 1 ㅇ.

Zu dieser Art bringe ich vorläufig obiges Exemplar, obgleich sie nur von den Arn-Inseln bekannt ist. Das Tier gehört zu der aenea-Gruppe, hat aber wie die Walkersche Art braunrote Schenkel und eine nackte Fühlerborste. Die Stirne ist fast ganz mattschwarz, nur vorn 
und hinten an den Ecken fettig glänzend, der mattschwarze Mittelflecken ist hier also viel mehr ansgedehnt als bei aenea. Untergesicht, Backen, Rüssel und Taster braunrot. Der Thorax ist grauschwarz, überall kurz schwarzbraun behaart; eine heller behaarte Mittelstrieme ist also nicht erkennbar, wohl fallen bei vorderer Ansicht 3 schwärzliche Längslinien auf, von welchen die mittlere sehr schmal ist. Hinterleib stärker glänzend als der Thorax, dunkel elzgrün.

In der Anm. bei Hendel (Abh. K. K. Zool. Bot. Ges. Wien, VIII, 1914, p. 52) sind longivitia und imitans z. T. verwechselt. Longivitta, von den Aru-Inseln ist die Art mit rostfarbenen Schenkeln und wurde von Walker in den Proc, Linn. Soc. III, p. 115 beschrieben.

$$
\text { Pseudepicausta Hend. }
$$

Pseudepicausta geniculata v. d. Wulp.

Suban Ajan, Juli.

Naupoda Ost. Sack.

Naupoda strigifera n. sp. Taf. II, Fig. 13. Muara Sako, October.

Stirne gelbbraun, etwas marmoriert, vorn mit der Spur einer dunkleren Querbinde; Periorbiten glänzend schwarz; jederseits 2 Orbitalborsten vorhanden. Die Stirne ist etwas breiter als ein Auge, parallelrandig. Wurzelglieder der Fühler rotgelb, das 3te Glied braun, die Borste kurz gefiedert. Untergesicht dunkelbraun, ausgehöhlt, vorn etwas vorstehend; Praelabrum gross, glänzend schwarz, Rüssel schwarzbraun; Hinterkopf nicht besonders gewölbt, d.unkelbraun, am Oberrande schmal gelb. Thorax samt Schildchen glänzend schwarz, mit kurzer, dunkler, braunschimmernder Behaarung. Schildchen mit ca. 8 schwarzen Randborsten. Der sehr kurze Hinterleib glänzend schwarz, kurz behaart, vorn dunkel, hinteu weisslich. Flügel an der Wurzel schwach gebräunt, was sich verwaschen bis zur Mitte der oberen Basalzelle erstreckt; in der Mitte mit einer schmalen, wenig gebogenen dunkelbraunen Querbinde, welche sich vom Vorder- bis zum Hinterrande erstreckt und die beiden Queradern berührt; überdies ein dunkles Querstrichelchen zwischen der 1 ten und 2 ten Längsader. Die kleine Querader liegt deutlich jenseits der Mitte der länglich rautenförmigen Discoidalzelle; hintere Basalzelle mässig gross, der sie unten distal abschliessende Abschnitt der 5ten Längsader kurz, queraderähnlich, kürzer als die Querader der Analzelle. Hüften schwarzbraun, die Vorderhüften an der Spitze schmal gelblich. Schenkel und Vorderschienen dunkelbraun, die hinteren Schienen gelbbraun, Vordertarsen schwarzbraun, die hinteren gelbweiss. Körperlänge $2,5-3 \mathrm{~mm}$; Flügellänge $3-4 \mathrm{~mm}$.

Diese Art dürfte $N$. punctifascia, Speis. aus Mittel-Afrika am ähnlichsten sein; diese unterscheidet sich durch stärker gefärbte Flügel, durch die grössere Länge der die hintere Basalzelle distal unten abschliessenden Querader, andere Beinfarbe u.s.w.

Die vor kurzem von Bezzi aus den Philippinen beschriebene $N$. unifasciata (Philipp. Journ. of Science. Vol. XII. $\mathrm{N}^{0}$ 3. Sec. D., 191\%, p. 140) ist offenbar gleichfalls, auch in der. Flügelfärbung, sehr ähnlich; sie unterscheidet sich u. a. durch gelbliche Taster, ganz gelbe vordere Tarsen, auch dadurch, dass nur jederseits eine Orbitalborste vorhanden ist. Die vorliegende Art hat deren 2, was für diese Gattung eine Ausnahme ist, doch kommen auch Arten vor, wo sie überhaupt fehlen; wie nach Bezzi bei platessa.

\section{Pterogenia Big.}

Pterogenia rubriceps n. sp. Taf. II, Fig. 14.

Air Njuruk, Dempu, August, 1 \%.

Kopf gelbrot, Stirne so breit wie ein Auge, parallelrandig, vorn gelbrot, in der Mitte etwas marmoriert, mit der Spur einer weisslichen Querbinde, im hinteren Teile dunkelbraun. Ocellenhöcker und Scheitelplatten glänzend schwarzbraun; die kurze Behaarung der Stirne 
schwarz. Von Kopfborsten sind nur die relativ kurzen inneren Scheitelborsten vorhanden. Wurzelglieder der Fühler gelbrot, das 3te Glied braun, die Borste nur an der Wurzel pubeszent. Hinterkopf oben schwarz mit schmalem gelben Rande, unten gelb. Fühlergruben weiss bereift, von einander getrennt. Wangen und Backen gerunzelt. Rüssel schwarzbraun, Taster gelbrot, an der äussersten Spitze verdunkelt. Thorax und Schildchen glänzend schwarz, punktiert, mit schwarzer Behaarung. Brustseiten schwarz, z. T. hellbraun bestäubt, die Behaarung dunkel. Hinterleib dunkel stahlblau, z. T. purpurn, namentlich in der Mitte, mit dunkelbrauner Behaarung, in der Mitte nicht gekielt. Hüften schwarz, Vorderschenkel an der Wurzel, unten bis zur Mitte, schwärzlich, weiterhin gelbrot; Mittelschenkel schwarzbraun, hinten mit rötlichem Längsstreifen; Hinterschenkel schwarz; Vorderschienen schwarz; Mittel- und Hinterschienen schwarz, in der Mitte breit rötlich; alle Tarsen schwarzbraun mit gelbweissem Metatarsus. Flügel mit schwarzbrauner Zeichnung, an der Wurzel mit mehreren dunklen Fleckchen, in der Mitte mit breiter Querbinde, welche die kleine Querader enthălt, unten mit einem vorderen Zipfel die $5^{\text {te }}$ Längsader weit überschreitend; die hintere Querader dunkel gesäumt, weiterhin mit mehreren länglichen Flecken in der Spitzenhälfte. 1te Hinterrandzelle am Ende kaum etwas erweitert; kleine Querader in der Mitte der Discoidalzelle. Schwinger schwarz mit hellbraunem Stiel; Schüppchen dunkel.

Körperlänge $5,5 \mathrm{~mm}$; Flügellänge $6 \mathrm{~mm}$.

Hendels Tabelle (Abhandl. K. K. Zool. Bot. Gesellsch. Wien. VIII, 1914, p. 304) führt auf $\mathrm{N}^{0}$ 3. Glabella unterscheidet sich $\mathrm{u}$. a. durch gelbe Flügelwurzel, fuliginosa durch lang gefiederte Arista. Glabra Walk. hat eine gelbe Hinterleibsbinde, divisa und truncatula haben helle Schwinger. Von allen ist die neue Art auch durch den hell gelbroten Kopf verschieden. Meiner atrata von Simalur sieht sie gleichfalls ähnlich, auch diese hat jedoch einen fast ganz schwarzen Kopf, eine langgefiederte Fühlerborste u. s. w.

Rhadinomyia Schin.

Rhadinomyia orientalis Schin.

Piek von Korintji, August; Gunung Singalang, Juni.

\section{TRYPETINAE.}

A nastrepha Schin.

Anastrepha extranea de Meij.

Air Njuruk, Dempu, August.

Colobostroter Enderl.

Colobostroter pulchralis Enderl.

Suban Ajam, Juli.

In beiden Stücken zeigt der Thorax 2 weit getrennte schwarze Längslinien, welche hinten auf die Seitenecken des Schildchens übertreten.

Ptilona v. d. Wulp.

Ptilona brevicornis v. d. Wulp.

Air Njuruk, Dempu, August; Suban Ajam, Juli.

Ptilona nigriventris Bezzi.

Pasumah Estate (Palembang), August.

Beim $\sigma^{7}$ ist der $2^{\text {te }}$ Hinterleibsring bisweilen nur am Vorderrande, bisweilen fast ganz gelb.

Rioxa Walk.

Rioxa sexmaculata v. d. Wulp.

Kep. Alüang. 


\section{Acanthoneura Macq.}

Acanthoneura Dunlopi v: d. Wulp.

Air Njuruk, Dempu, August; Suban Ajam, Juli.

Acanthoneura (Acinia ap. Enderlein) stellata Macq.

Muara Sako, October.

- Das Exemplar zeigt auf der Flügelfläche ein paar kleine Fleckchen weniger als in Enderlein's Figur (Zool: Jahrb. Abt. Syst., Bd. .31, 1911, p. 433).

Frey erwähnt diese bis zu den Philippinen verbreitete Art auch von Ceylon (Finsk. Vet. Soc. Förh. LIX, 191\%, Afd. A. N ${ }^{0} 20$, p. 19).

Poecillis Bezzi.

Poecillis judicanda Bezzi.

Suban Ajam, Juli.

Das Schildchen ist schwarz, mit breitem, gelbem Hinterrand. Es wurden 13 우 dieser bisher nur aus Bengalen bekannten Art erbeutet, sodass die Frage Bezzi's, ob hier vielleicht das ㅇ von Chaetellipsis paradoxa Bezzi vorliegen könnte, eine offene bleibt.

\section{Chelyophora Rond.}

Chelyophora bilineata de Meij.

Suban Ajam, Juli; Kep. Alüang.

Die gelbe Thoraxzeichnung ist bisweilen etwas ausgedehnter, sodass z. B. auf dem Schildchen 2 bis zur 'Spitze verlaufende Längslinien von der Basis ausgehen. Die anale Flügelecke ist bei den sumatranischen Stücken gleichmässiger gefärbt als bei der javanischen Type, sodass auch die Analzelle einen gelben Ton zeigt.

\section{PSILINAE.}

\section{Strongylophthalmyia Hend.}

Strongylophthalmyia lutea de Meij.

Air Njuruk, Dempu, 1400 M., August, 1 ㅇ.

Die Type von Java ist offenbar nicht ganz ausgereift. Das hier vorliegende Stück ist dunkler, von den Fühlern ist das $3^{\text {te }}$ Glied ganz schwarz, ein grosser Mittelfleck der vorderen Thoraxhälfte ist glänzend schwarz und erstreckt sich seitlich bis zu den Schulterbeulen, hinten bis. zur Quernaht, auf den Flügeln sind die Binde und die Spitze stärker verdunkelt.

Strongylophthalmyia polita de Meij.

Suban Ajam, $\%$.

Strongylophthalmyia metatarsata $\mathrm{n}$. sp.

Air Njuruk, Dempu, 1400 M., August, 2 \&

Kopf glänzend schwarz. Fühler schwarzbraun, weisslich behaart, mit nackter Borste; Taster und Rüssel schwarz. Thorax glänzend schwarz, mit schwarzer Behaarung; Hinterleib glänzend purpurschwarz. Flügel mit 3 schwarzbraunen Binden, die 1te, am wenigsten vollständige geht von der Spitze der 1ten Längsader nach unten, umfasst auch die kleine Querader, überschreitet die $4^{\text {te }}$ Längsader nur wenig; die 2te verläuft als vollständige Binde von der Spitze der 2ten Längsader zum Hinterrand, sie enthält die hintere Querader; die $3^{\text {te }}$ ]iegt an der Flügelspitze. Die Spitze der 2ten Längsader liegt jenseits der hinteren Querader. Schwinger. weiss mit schwarzem Stiele. Beine schwarz, die Trochanteren gelblich, die Meta- 
tarsen der Vorderbeine jin der Wurzelhälfte, die der Mittelbeine ganz, nebst einem Teile des $2^{\text {ten }}$ Gliedes, die der Hinterbeine mit Ausnahme der Spitze gelbweiss. Flügellänge $4 \mathrm{~mm}$.

Strongylophthalmyia fasciolata n. sp.

Suban Ajam, Juli, 2 웅.

Kopf glänzend schwarz; Fühler braungelb, das dritte Glied am Rande verdunkelt, die Borste nackt; Taster gelbbraun, an der Spitze dunkler. Thorax schwarz, oben wenig glänzend und fein runzelig, die Behaarung viel spärlicher als bei der vorhergehenden Art, nur die Dorsocentral- und Acrostichalreihen vorhanden. Brustseiten glänzend sčhwarz. Hinterleib desgleichen, mässig glänzend. Flügel mit 2 wenig starken Binden; die von der Spitze der $2^{\text {ten }}$ Längsader ausgehende erreicht den Hinterrand kaum, auch die an der Flügelspitze liegende ist verwaschen; kleine Querader schmal dunkel gesäumt. Spitze der 2 ten Längsader beträchtlich jenseits der hinteren Querader liegend. Letzter Abschnitt der 4 ten Längsader am Anfang deutlich aufwärts gebogen. Schwinger weiss mit dunkelbraunem Stiel. Vorderbeine, auch die Hüften, braungelb, die Schenkel und Schienenwurzel dunkler braun, an den Mittelbeinen sind die Hüften, die Endhälfte der Schienen und die Metatarsen gelb, an den Hinterbeinèn nur letztere.

Körperlänge $6 \mathrm{~mm}$; Flügellänge $4,5 \mathrm{~mm}$.

Die 4 mir aus Java, bzw. Sumatra bekannten schwarzen Arten mit gebänderten Flügeln unterscheiden sich wie folgt:

1. Thoraxrücken fein runzelig, nicht glänzend ....2.

$$
\text { " glänzend schwarz .... 3. }
$$

2. Vorderhüften schwarz, Flügel an der Spitze mit 3 getrennten schwarzen Fleckchen Str. tripunctata de Meij.

Vorderhüften grösstenteils rotgelb; Flügelspitze verwaschen verdunkelt Str. fasciolata n. sp.

3. Vorderhüften schwarz .... Str. metatarsata n. sp.

» grossenteils gelb.... Str. maculipennis Hend.

Strongylophthalmyia nigricoxa de Meij.

Air Njuruk, Dempu, August, : einige $q$ ㅇ.

\section{CHLOROPINAE.}

Formosina Beck.

Formosina atrata n. sp.

Suban Ajam, Juli.

Von breiter Gestalt und glänzend schwarzer Farbe. Kopf matt schwarzbraun, das Scheiteldreieck glänzend schwarz, bis zum vorderen Stirnrande reichend; Fühler dunkelbraun, das

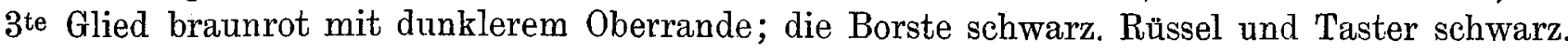
Backen sehr schmal. Augen längsoval. Thorax, Schildchen und Hinterleib glänzend schwarz, der Thoraxrücken sehr kurz schwarz behaart. Schildchen oben abgeflacht, unten am Rande mit 2 Endborsten. Flügel glashell. Randader jenseits der 1 ten Längsader mässig verdickt. Schwinger gelb. Beine schwarz, an den vorderen Beinen der Metatarsus braungelb, an den Hinterbeinen die 3 ersten Glieder gelb. Körper- und Flügellänge $3,5 \mathrm{~mm}$.

Trotzdem diese Art am Thorax, auch am Schildchen nichts Gelbes aufweist, gehört sie im übrigen doch durchaus in diese Gattung.

\section{Chalcidomyia de Meij.}

Chalcidomyia laticornis de Meij.

Air Njuruk, Dempu, August. 
Chlorops $\mathrm{Mg}$.

Chlorops incisa de Meij.

Suban Ajam, Juli.

Diese Art war bis jetzt nur von Krakatau bekannt.

Loxotaenia Beck.

Loxotaenia halterata n. sp.

Muara Sako, October.

Meiner $L$. gracilis äusserst ähnlich, sodass es genügt die Unterschiede anzugeben. Der Thorax ist mehr bronzefarben, nicht glänzend schwarz; der Hinterleib vorn glänzend bronzefarben, hinten schwarz. Die Flügel sind etwas gebräunt, der letzte Abschnitt der 4ten Längsader weniger unscheinbar als bei gracilis; die Schwinger sind schwarzbraun, während sie bei gracilis weiss sind. Körper- und Flügellänge etwas mehr als 2 mm.

Meroseinis de Meij.

Meroscinis lucidifrons $\mathrm{n} . \mathrm{sp}$.

Suban Ajam, Juli.

Stirne schwarz, etwas fettig glänzend, das Scheiteldreieck die Fühlerwurzel nicht erreichend, glatt, sehr stark glänzend; die Behaarung der Stirne schwarz. Fühler rotgelb, das 3te Glied am Oberrande schmal verdunkelt; die Borste schwarz, kurz behaart. Untergesicht, die schmalen Backen, Rüssel und Taster schwarz. Thorax und Schildchen schwarz, mässig glänzend, punktiert, mit dunkler, gelb schimmernder Behaarung; das Schildchen lang dreieckig, fast so lang wie der Thoraxrücken, jederseits mit 3 starken gelben Borsten, die Endborsten am längsten. Brustseiten glänzend schwarz, zerstreut kurz weiss behaart. Hinterleib glänzend schwarz, die Basis breit durchscheinend gelb. Fühler glashell, an der Wurzel etwas gelblich, die 2te Längsader nicht lang, etwas gebogen, der $2^{\text {te }}$ und 3te Abschnitt des Vorderrandes deswegen von gleicher Länge; 3te und $4^{\text {te }}$ parallel. Letzter Abschnitt der $4^{\text {ten }}$ Längsader gerade. Sehwinger gelb. Beine ganz gelb, nur das letzte Tarsenglied dunkelbraun. Körperlänge 2,5 mm; Flügellänge $2 \mathrm{~mm}$.

Becker's Tabelle (Ann. Mus. Nation. Hungar., IX, 1911, p. 88) führt auf elegantula und recta. Elegantula unterscheidet sich durch viel grösseres, weniger glattes Scheiteldreieck, dunkleres $3^{\text {tes }}$ Fühlerglied, relativ kürzeres Schildchen mit dunklen Borsten, von welchen die seitlichen relativ kürzer sind, durch an der Basis erweiterte 1te Hinterrandzelle, weil der letzte Abschnitt der $4^{\text {ten }}$ Längsader an der Basis etwas aufgebogen ist. Recta ist kleiner, hat ein viel kürzeres Schildchen; der $2^{\text {te }}$ Vorderrandsabschnitt ist bedeutend Iänger als der $3^{\text {te }}$, weil die $2^{\text {te }}$ Längsader länger und nur an der Spitze gebogen ist. $M$. quadriseta de Meij. ('lijdschr. v. Entom., LVI, 1913, p. 296) scheint mir doch nur zu den Varianten von elegantula zu gehören.

Meroscinis sumatrensis n. sp.

Sungai Kuimbang, September.

Von schmaler Gestalt. Das sehr grosse, bis zu den Fühlern reichende Scheiteldreieck glänzend grünlich schwarz, gelb behaart; Ocellarborsten relativ stark; die winzigen übrigen Stirnteile matt dunkelbraun. Fühler ganz gelb, Borste dunkelbraun, dünn, kurz behaart. Das kurze Untergesicht unter den Fühlern glänzend schwarzbraun, der Mundrand gelb. Rüssel und Taster braun. Thorax länger als breit, glänzend schwarz, punktiert, ziemlich dicht gelb behaart. Brustseiten glänzend schwarz, wenig behaart. Schildchen länglich dreieckig, ausser den 2 langen gelben Endborsten jederseits noch mit 2 Borsten von halber Länge. Hinterleib langgestreckt, glänzend schwarz, glatt, an der Wurzel in der Mitte etwas rötlich, flachgedrückt, mit zerstreuter oben dunkler, am Rande heller Behaarung. Flügel kaum etwas bräunlich; 3te und $4^{\text {te }}$ Längsader parallel, der letzte Abschnitt der $4^{\text {ten }}$ im Anfang etwas nach 
oben geschwungen. Beine gelb, Vorderschenkel mit der Spur eines dunklen Querringes, namentlich ein dunkler Wisch an den dem Körper zu- und abgewandten Seiten deutlich; Vorderschienen etwas gebogen, schwarz, nur an der Spitze und an der Innenseite etwas gelb. Mittelschenkel vorn mit kleinem, Hinterschenkel mit grösserem strichförmigen Wisch; diese Schenkelwische beim $2^{\text {ten }}$ Stück nicht erkennbar. Tarsen ganz gelb, oder, beim $2^{\text {ten }}$ Stück, die Vordertarsen gebräunt, an den hinteren die beiden Endglieder etwas verdunkelt. Körperlänge $4 \mathrm{~mm}$; Flügellänge $3 \mathrm{~mm}$.

M. sumatrensis sieht der vorhergehenden ähnlich, ist aber grösser, das Scheiteldreieck ist deutlich grünlich und weniger glatt; die seitlichen Schildchenborsten sind kürzer. Von der sehr ăhnlichen elegantula ist sie, ausser durch die Beinfärbung, durch die ganz hellen Fühler und durch das Schildchen mit den 6 gelben Borsten zu unterscheiden.

Meroscinis Meyerei Beck.

Muara Sako, October.

Meroscinis scutellata de Meij.

Suban Ajam, Juli.

Dactylothyrea de Meij.

Dactylothyrea armata n. sp:

Air Njuruk, Dempu, August.

Sehr ähnlich meiner $D$. hyalipennis, aber verschieden durch ganz gelbrote Hühler; die vordere Hinterleibshälfte ist ganz gelb. Die Flügel sind etwas gebräunt, weniger glashell als bei der anderen Art; die Schildchendornen sind relativ stärker entwickelt, die hinteren deutlich länger als das Schildchen, die seitlichen gebogen, so lang wie das Schildchen an der Basis breit. Körperlänge $4 \mathrm{~mm}$; Flügellänge $3 \mathrm{~mm}$.

Oscinella $\cdot$ Beck.

Oscinella obscuripennis n. sp.

Muara Sako, October.

Von schmaler Gestalt. Stirne schwarzbraun, vorn sehr schmal und wenig deutlich rötlich. Scheiteldreieck gleichfalls schwarzbraun, mässig glänzend aber nicht besonders scharf von der übrigen Stirnfläche abgetrennt. Stirnborsten relativ deutlich. Fühler schwarzbraun, das runde $3^{\text {te }}$ Glied an der Innenseite mit einem roten Flecken nahe der Basis. Borste schwarz, kurz behaart. Untergesicht fast gerade, grauweiss bestäubt. Backen sehr schmal, vorn etwas rötlich. Rüssel und Taster schwarz. Hinterkopf dunkelbraun, unten wie die Backen mit weisslichem Schimmer. Thorax schwarz, mässig glänzend, mit schwachen metallischen Reflexen, dünn kupferbraun bereift, mit zerstreuter schwarzer Behaarung. Brustseiten schwarz, an der Flügelwurzel mit einer breiten weiss bestäubten Querbinde, desgleichen die Gegend über den Vorderhüften weiss bestäubt. Schildchen bläulich schwarz, mit 4 Borsten, die âusseren schwach. Hinterleib glänzend schwarz. Flügel grösstenteils ziemlich stark gebräunt, die Costalzelle, die Wurzel der Discoidalzelle und ein breiter Hinterrandsteil glashell,

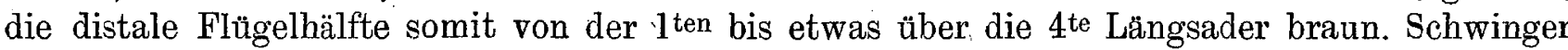
gelbweiss mit dunklerem Stiele. Beine schwarz, Vorderhüften dicht weiss bestäubt; an den hinteren Beinpaaren der Metatarsus und die äusserste Spitze der Schienen rotgelb. Körperund Flügellänge $3,5 \mathrm{~mm}$.

\section{GEOMYZINAE.}

Amphoroneura de Meij

Amphoroneura obscura de Meij.

Air Njuruk, Dempu, August. 


\section{LAUXANIINAE.}

Lauxania orientalis Wied.

Lauxania Lotz.

Muara Sako, October.

Lauxania picta de Meij.

Fort de Kock, November; Air Njuruk, Dempu, August.

Lauxania immaculata de Meij.

Suban Ajam, Juli; Sungai Kumbang, September.

Lauxania quinquevittata de Meij.

Sungai Kumbang, September; Piek von Korintji, August.

\section{GELYPHINAE.}

Celyphus Dalm.

Celyphus oblectus Dalm.

Palembang (Knappert leg.)

Celyphus aurora Karsch.

Palembang (Knappert leg.)

Spaniocelyphus Hend.

Spaniocelyphus trigonalis de Meij., subsp. extensus subsp. n.

Palembang (Knappert leg.).

Das Exemplar sieht dem von Kalung (Padangsche Bovenlanden, Sumatra; Studien X, p. 94) ganz ähnlich. Auch in den Flügeln ist einige Verschiedenheit mit dèr Type von Simalur, sie sind an der Spitze weniger breit und etwas länger; der $4^{\text {te }}$ Abschnitt der $4^{\text {ten Längs- }}$ ader ist dadurch relativ länger, bei der Type etwas kürzer als der vorletzte, deshalb wenigstens als subspecies: extensus zu trennen. 


\section{TAFELERKLÄRUNG.}

\section{TAFEL II.}

Fig. 1. Dicranomya signata n. sp. Flügel.

" 2. Limnobia stigmosa n. sp.

" 3. Libnotes tripunctata n. $\mathrm{sp}$

4. Acyphona punctulata n. sp.

" 5. Gnophomyia fascipennis n. sp. "

" 6. Epiphragma Kerberti n. sp.

" \%. Agastomyia albitarsis n. sp.

" 8. Sphaerionotus curtipennis n. sp., von der Seite.

" 9. Rosapha variegata n. sp. Flügel.

" 10. Chrysopilus lupinus Ost. Sack. Flügel.

, 11. Tachytrechus argentatus $\mathrm{n}$. sp.

" 12. Nerius tinctipennis n. sp.

"13. Naupoda strigifera n. sp.

" 14. Pterogenia rubriceps n. sp 


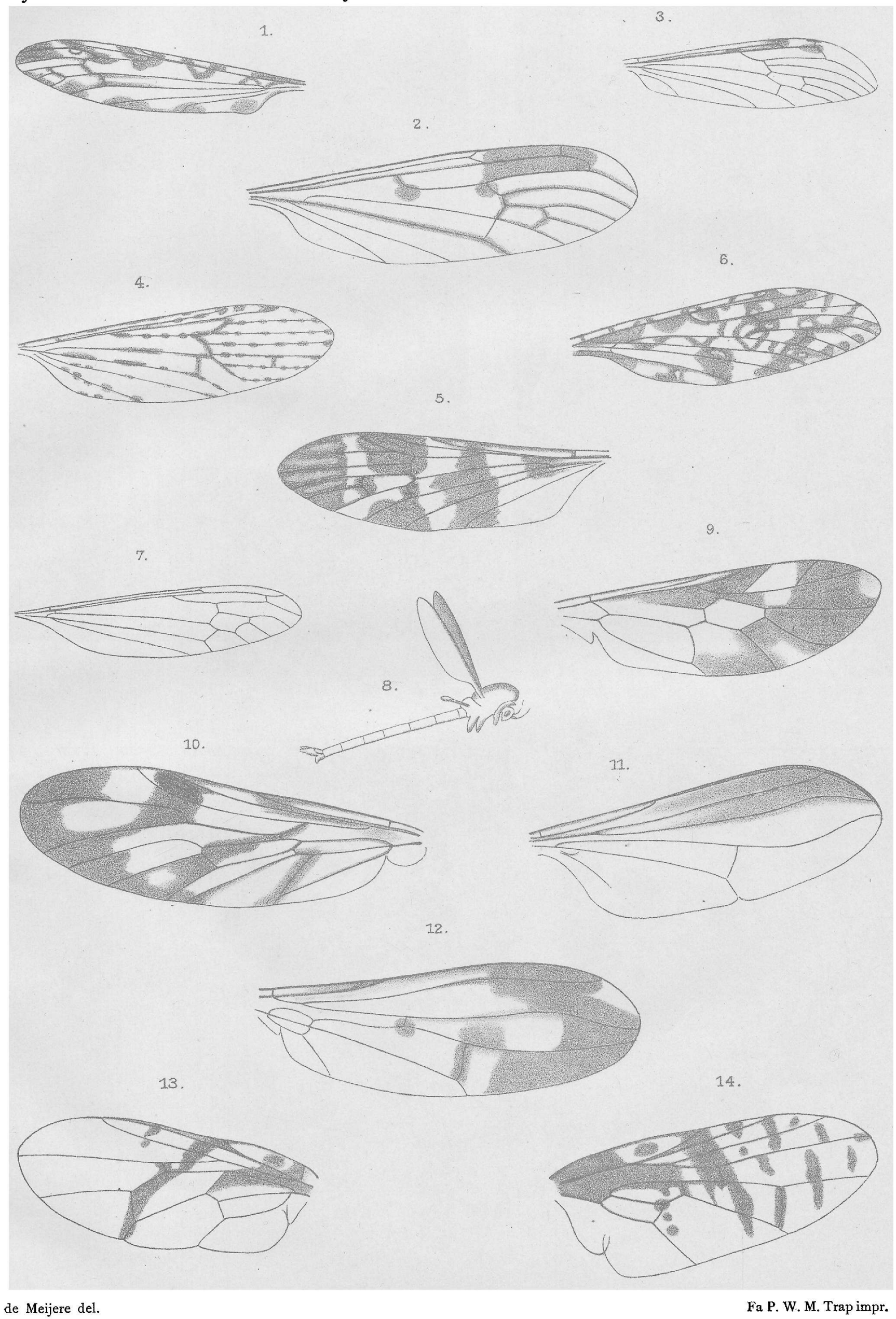

